\title{
Solute transport in random composite media with uncertain dispersivities
}

\author{
Aronne Dell'Oca ${ }^{\mathrm{a}, *}$, Monica Riva ${ }^{\mathrm{a}}$, Philippe Ackerer ${ }^{\mathrm{b}}$, Alberto Guadagnini ${ }^{\mathrm{a}}$ \\ ${ }^{a}$ Dipartimento di Ingegneria Civile e Ambientale, Politecnico di Milano, Piazza L. Da Vinci 32, Milano 20133, Italy \\ ${ }^{\mathrm{b}}$ Hydrology and Geochemistry Laboratory - LHyGeS, Strasbourg University - CNRS, 67000 Strasbourg, France
}

\section{A B S T R A C T}

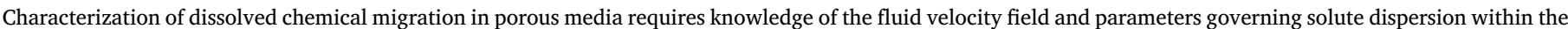

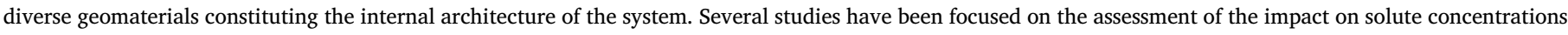

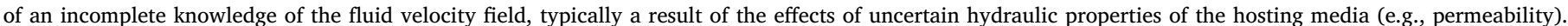

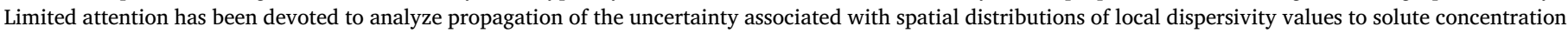

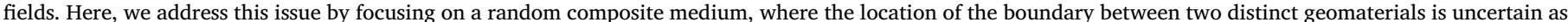

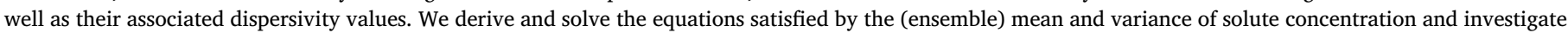

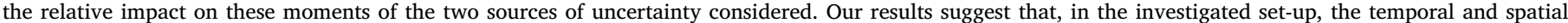

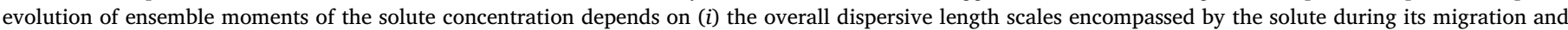
(ii) the actual sequence of the materials traversed by the solute.

\section{Introduction}

Heterogeneity of natural subsurface systems, as well as data scarcity and measurement errors, have promoted the adoption of stochastic approaches to investigate flow and transport features in porous media under multiple sources of uncertainty. In this context, a wide range of stochastic approaches is available including, e.g., techniques based on numerical Monte Carlo simulations, Stochastic Finite Element (Ghanem and Spanos, 1991), Moment Equations (Guadagnini et al., 2003; Morales et al., 2006a,b; Neuman, 1993; Winter et al., 2002 and references therein], Probability Density Function (Boso and Tartakovsky, 2016; de Barros and Fiori, 2014; Dentz and Tartakovsky, 2010 and reference therein), and/or Probabilistic Collocation (Li and Zhang, 2007) methods.

When considering random heterogeneity in the spatial distribution of parameters governing flow and transport in porous media, much attention has been devoted to the analysis of the importance of loghydraulic conductivity heterogeneity, dispersion being usually considered as (deterministically) uniform at a given scale of interest. In this context, it is important to distinguish between dispersion and spreading processes, and their links with the scale of interest at which solute transport is modeled (see e.g., Kitanidis, 2017 and references therein). Consistent with the approach employed in several previous studies (e.g., Fernàndez-Garcia et al., 2009; Godoy et al., 2018; Moslehi et al., 2016; Vishal and Leung, 2018; Xu and Meakin, 2013), our work rests on the following concepts: "Dispersion is often used as synonym for spreading or rate of spreading, as in the spreading of a large plume. This is not the meaning of this term as used in this work. Instead, by dispersion we mean the

\footnotetext{
* Corresponding author.

E-mail address: aronne.delloca@polimi.it (A. Dell'Oca).
}

transport mechanism that describes fluxes not captured by the velocity and the concentration that are resolved at some finite scale. In simple terms, the velocity and concentrations we use in our conceptual or mathematical models are smooth versions of the actual velocity and concentration that are much more variable; the omitted fluctuations collectively have an effect that we call dispersion. Clearly, the dispersion mechanism cannot be discussed without having a fixed idea of the scale of resolution we are concerned with." (quoted from Kitanidis, 2017). It is then natural to expect that there is uncertainty in the intensity of the dispersion process at the selected scale of representation of the solute transport phenomena. Indeed, theoretical developments and experimental evidences associated with diverse scales of investigation show that dispersivity values should be considered as a spatially variable (random) process, (Chaudhuri and Sekhar, 2005; Coelho et al., 1997; de Barros and Rubin, 2011; Gist et al., 1990; Godoy et al., 2018; Kuo et al., 1999; Woods et al., 2003; Xu and Meakin, 2013).

At the laboratory scale, dispersivity is typically referred as local dispersivity. The latter is mainly related to the particle size distribution of a porous material e.g., Coelho et al. (1997), Gist et al. (1990), $\mathrm{Xu}$ and Meakin (2013). A detailed review of experimental findings encompassing analyses of more than 700 solute breakthrough curves collected under steady-state flow conditions has been performed by Kuo et al. (1999) and highlights the strong correlation between local dispersivity values and soil texture and its spatial variability.

At larger (field-regional) scales, dispersivity is typically denoted as macrodispersivity and is mainly related to spatial variability of geomaterials and the associated hydraulic conductivity distributions. Here, it is important to properly assess the scale of interest. When considering plume-scale macrodispersivity (e.g., Gelhar and Axness, 1983; 
Zech et al., 2015, and references therein) the scale of interest evolves with the size of the plume and the investigated process is the rate of spreading of the solute cloud. Note that the plume-scale macrodispersivity is basically a deterministic quantity under ergodic conditions (see e.g., de Barros and Rubin, 2011 and references therein). Otherwise, considering a finite (and fixed) scale of resolution requires relying on upscaled or block-scale macrodispersivity, whose role is that of rendering the unresolved variability associated with lower resolution scales (see e.g., de Barros and Rubin, 2011). Chaudhuri et al. (Chaudhuri and Sekhar, 2005) analyzed the effects on the plume-scale macrodispersion of random spatial heterogeneity of local dispersivity and hydraulic conductivity through a first-order approximation technique. The work of de Barros and Rubin (2011) highlighted the need to treat the block-scale dispersion coefficient as a random space function, in order to accommodate for the uncertainty in the solute dispersion process rendered at a given scale under non-ergodic conditions. The work of de Barros and Dentz (2016) extended the analysis by distinguishing between effective and ensemble block-scale dispersion coefficients and evaluating the associated uncertainty. Similarly, Woods et al. (2003) rely on a volumeaveraging approach and indicate that the ensuing dispersion coefficient is subject to diverse degrees of uncertainty as a function multiple factors, including, e.g., the size of the averaging-volume (that could be interpreted as the finite scale of interest for the resolution of solute transport) and the statistical properties of the hydraulic conductivity field. Recently, Godoy et al. (2018) applied a geostatistical approach to evaluate macro-dispersion coefficients and to reproduce transport features in a tropical soil formation considering heterogeneous distribution of (local scale) hydraulic conductivities and dispersivities.

Despite these evidences, the impact of spatial heterogeneity of dispersivity on main features of transport within porous domains composed by multiple geomaterials which can be randomly distributed across the system is still largely unexplored. We tackle this issue by formulating and solving equations satisfied by the first two (ensemble) moments of concentration of a nonreactive dissolved chemical migrating in a porous domain composed by diverse geomaterials, each associated with a random local dispersivity, and separated by an internal boundary whose location is uncertain.

The impact of uncertainty in the spatial distribution of geomaterials constituting the internal architecture of an aquifer system and eventually associated with a random spatial variability of hydraulic conductivity has been previously analyzed in the context of fully saturated groundwater flow by relying on equations satisfied by the first and second statistical moments of hydraulic head and Darcy' fluxes (e.g., Guadagnini et al., 2003; Tartakovsky and Guadagnini, 2004; Winter et al., 2002; Winter and Tartakovsky, 2000 and references therein). These works show that conceptualizing the domain as a random composite has the benefit of replacing a porous system characterized by a large spatial variability of hydraulic conductivities, as reflected by their variance, with a relatively tractable setting where one considers the flow and transport domain as composed by a set of disjoint geomaterials. These are separated by an eventually random boundary and each geomaterial of the composite can be associated with a hydraulic conductivity distribution of low to moderate variance. The latter requirement is key to ensure convergence of perturbation-based approaches which are typically at the heart of Moment Equations (MEs) methods of the kind we assess in this study.

We note that the ME approach has been limited to the analysis of solute transport (as described through a classical Advection Dispersion Equation at a given scale) in porous formations filled with a single geomaterial where a spatially heterogeneous fluid flow field takes place, as driven by the spatial heterogeneity of hydraulic conductivity e.g., Berkowitz et al. (2006), Cushman and Ginn (1993), Morales et al. (2006a,b), Rubin (2003) and references therein.

As an exemplary showcase, we solve our MEs by focusing on a domain formed by a one-dimensional domain filled by two adjacent geological units, i.e., a coarse (C) and a fine (F) medium separated by an interface perpendicular to the mean flow direction. We focus on this relatively simple configuration (i) in order to facilitate understanding of the impact of diverse sources of uncertainty on the leading statistical moments of solute concentrations and (ii) in the light of the possibility of employing a one-dimensional conceptualization of the system (typically considered as filled with a unique geomaterial) for the interpretation of laboratory and field scale experiments (see, e.g., Fiori et al., 2017; Gramling et al., 2002; Levy and Berkowitz, 2003; Porta et al., 2015). Indeed, the setup we analyze has been studied by several authors (e.g., Berkowitz et al., 2009; Kuo et al., 1999; Leij and van Genuchten, 1995; Sternberg, 2004 and references therein) using different settings of fine and coarse materials.

In this framework, and to the best of our knowledge, Berkowitz et al. (2009) provides the only experimental results documenting asymmetry in solute breakthrough curves (BTCs) associated with (i) a coarse to fine arrangement (hereafter denoted as C-F), where a coarse material precedes a fine material along the direction of flow; and (ii) a fine to coarse arrangement (hereafter termed F-C). While these experimental results are not consistent with the traditional Fickian behavior of solute transport in columns, the reasons underpinning the documented discrepancy are still under discussion (see, e.g., the studies of Appuhamillage et al., 2010; Alvarez-Ramirez et al., 2014; Cortis and Zoia, 2009; Zhang et al., 2010).

Our study aims at investigating the effect of the lack of knowledge on (i) values of local dispersivities and (ii) location of the boundary interface on our ability to quantify the space- time evolution of statistical moments of solute concentrations. For this purpose, we rely on the classical Advection Dispersion Equation (ADE). In this context, the local dispersivity value of each geomaterial embeds the impact of the unresolved (i.e., below the accessible finite scale of resolution of transport phenomena, coinciding here with the length of each distinct geomaterial) velocity and concentration fluctuations onto the ensuing concentration at the resolved scale, which is here assumed to be described through the ADE model. Taking into account heterogeneity in the dispersion process (here mapped onto dispersivity coefficients) at accessible scales of resolution could then affect the spreading behavior of the whole solute plume traveling through the system, this picture being conceptually akin to the case in which heterogeneity of hydraulic conductivity is recognized to exert an influence at a given scale of resolution (see e.g., Le Borgne et al., 2010; Morales et al., 2006a,b). Lack of data and measurement errors that are typically affecting flow and transport in porous media are then tackled within a stochastic approach (as rendered through MEs in this study) to treat diverse sources of uncertainty.

The rest of the manuscript is organized as follows. The set-up of the problem is described in Section 2. We then derive MEs satisfied by solute concentrations evolving in the presence of uncertain geomaterial dispersivities. The resulting (statistical) moments of concentrations are either conditional to our knowledge of the location of the geomaterial interface or fully take into account randomness in its location. One of the most attractive aspects of the ME-based formulation, as opposed to fully numerical Monte Carlo simulations, is that it enables us to clearly elucidate the functional dependencies between the statistics of system parameters and of the concentration field, as described in Section 3 where our key results and main findings are illustrated and discussed. Conclusions are drawn in Section 4.

\section{Methodology}

\subsection{Problem set up and transport model}

We consider a one-dimensional domain of length $L^{*}$ composed by two geomaterials separated by a sharp interface located at $x^{*}=\beta^{*}$. The longitudinal local dispersivity, $\alpha_{L}^{*}$, of the system is represented as

$\alpha_{L}^{*}\left(x^{*}\right)=\alpha_{L_{1}}^{*} H\left(\beta^{*}-x^{*}\right)+\alpha_{L_{2}}^{*} H\left(x^{*}-\beta^{*}\right)$ 
where $H$ is the Heaviside function, $\alpha_{L_{1}}^{*}$ and $\alpha_{L_{2}}^{*}$ respectively being the local dispersivities of the two geomaterials. We take solute transport to be governed by the classical Advection Dispersion Equation (ADE), i.e.,

$\frac{\partial C^{*}\left(x^{*}, t^{*}\right)}{\partial t^{*}}+u^{*} \frac{\partial C^{*}\left(x^{*}, t^{*}\right)}{\partial x^{*}}-u^{*} \frac{\partial}{\partial x^{*}}\left(\alpha_{L}^{*}\left(x^{*}\right) \frac{\partial C^{*}\left(x^{*}, t^{*}\right)}{\partial x^{*}}\right)=0$

where $C^{*}\left(x^{*}, t^{*}\right)$ is solute concentration and $u^{*}$ is fluid velocity. Note that (1)-(2) rest on the Scheidegger dispersion model, other models being compatible with the approach we pursue. We also note that our analysis can be extended to cases where the ADE is employed to describe an upscaled (from a continuum to an increased continuum scale) solute concentration field, by, e.g., introducing block-scale dispersion coefficients, where local dispersivities within each geomaterial are also heterogeneous. Resident water is displaced by the instantaneous injection at the domain inlet (i.e., at $x^{*}=0$ ) of a small volume $V^{*}$ of solution with concentration $C_{0}^{*}$. Eq. (2) is then subject to the initial and boundary conditions

$C^{*}\left(x^{*}, t^{*}\right)=C_{0}^{*} \frac{V^{*} \delta^{*}\left(x^{*}\right)}{\phi} \quad$ for $\quad 0 \leq x^{*} \leq L^{*}, \quad t^{*}=0$

Here, $\phi$ is medium porosity assumed to be constant and deterministically known, resulting in a constant and deterministic fluid velocity.

We set the following boundary conditions

$\lim _{x^{*} \rightarrow 0^{+}}-\alpha_{L_{1}}^{*} \frac{\partial C^{*}\left(x^{*}, t^{*}\right)}{\partial x^{*}}=0 \quad \forall t^{*}>0$

$\frac{\partial C^{*}\left(x^{*}, t^{*}\right)}{\partial x^{*}}=0 \quad$ for $x^{*}=L^{*}, \quad \forall t^{*}>0$

Here, (4a) states that the dispersive component of transport could be neglected in comparison with the advective component at the inlet boundary. Note that, according to (4a) no solute mass leaves the domain from the inlet due to dispersive mechanisms.

Introducing the following dimensionless quantities

$x=\frac{x^{*}}{L^{*}} ; \quad t=\frac{t^{*}}{L^{*} / u^{*}} ; C_{x}=\frac{C^{*}}{C_{0}^{*}} ; \quad \delta=\frac{V^{*} \delta^{*}}{\phi} ; \beta=\frac{\beta^{*}}{L^{*}} ;$

$\alpha_{L}=\frac{\alpha_{L}^{*}}{L^{*}} ; \quad \alpha_{L_{i}}=\frac{\alpha_{L_{i}}^{*}}{L^{*}} ;$ with $i=1,2$

Eqs. (1)-(4) can be rewritten in dimensionless form as

$\alpha_{L}(x)=\alpha_{L_{1}} H(\beta-x)+\alpha_{L_{2}} H(x-\beta)$

$\frac{\partial C_{x}}{\partial t}+\frac{\partial C_{x}}{\partial x}-\frac{\partial}{\partial x}\left(\alpha_{L}(x) \frac{\partial C_{x}}{\partial x}\right)=0$

$C_{x}=\delta(x) \quad$ for $0 \leq x \leq 1, \quad t=0$

$\lim _{x \rightarrow 0^{+}} \alpha_{L_{1}} \frac{\partial C_{x}}{\partial x}=0, \quad \frac{\partial C_{x}}{\partial x}=0$ for $\quad x=1, \quad \forall t>0$

Here we denote $C_{x}=C(x, t)$ and dimensional time in (5) has been rescaled with respect to the characteristic advective time, $L^{*} / u^{*}$, the latter being a constant deterministic quantity in this study.

Our analysis considers the two local dispersivities $\alpha_{L_{1}}$ and $\alpha_{L_{2}}$ as well as the location $\beta$ of the interface between the two media as uncorrelated random variables, each characterized by a given probability density function, hereafter denoted as $p\left(\alpha_{L_{1}}\right), p\left(\alpha_{L_{2}}\right), p(\beta)$ for $\alpha_{L_{1}}, \alpha_{L_{2}}$ and $\beta$, respectively. On these bases, we illustrate the equations satisfied by the leading (statistical) moments of $C_{x}$ in the following sub-section.

\subsection{Moment equations for solute concentration}

The ensemble mean, $\langle\psi\rangle$, of a given quantity of interest $\psi$ associated with the system described in Section 2.1 can be evaluated as
$\langle\psi\rangle=\left.\int_{\Gamma_{\beta}}\langle\psi\rangle\right|_{\beta} p(\beta) d \beta$ with $\left.\langle\psi\rangle\right|_{\beta}=\left.\int_{\Gamma_{\alpha_{L_{2}}}} \int_{\Gamma_{\alpha_{L_{1}}}} \psi\right|_{\beta} p\left(\alpha_{L_{1}}\right) p\left(\alpha_{L_{2}}\right) d \alpha_{L_{1}} d \alpha_{L_{2}}$

Here, $\left.\langle\psi\rangle\right|_{\beta}$ denotes the expected value of $\psi$ conditional to a given position of the geomaterial interface, i.e., the expected value of $\psi$ when the location of the interface $\beta$ is known and only the two geomaterial local dispersivities are affected by uncertainty.

In Section 2.2.1, we derive the equations satisfied by the mean and variance of $C_{x}$ when $\alpha_{L_{1}}$ and $\alpha_{L_{2}}$ are uncertain while $\beta$ is given, Section 2.2.2 being devoted to the assessment of moments of $C_{x}$ when all quantities $\alpha_{L_{1}}, \alpha_{L_{2}}$ and $\beta$ are uncertain.

\subsubsection{Moment equations for solute concentration with known location of} geomaterial interface

We make use of the following decomposition

$\alpha_{L_{i}}=\left\langle\alpha_{L_{i}}\right\rangle+\alpha_{L_{i}}^{\prime}$ with $\left\langle\alpha_{L_{i}}\right\rangle=\int_{\Gamma_{\alpha_{L_{i}}}} \alpha_{L_{i}} p\left(\alpha_{L_{i}}\right) d \alpha_{L_{i}}$

where $\alpha_{L_{i}}^{\prime}(i=1,2)$ is the fluctuation of $\alpha_{L_{i}}$ with respect to its mean, $\left\langle\alpha_{L_{i}}\right\rangle$. Considering (7)-(8), the second of (9) and making use of (10), the mean solute concentration, $\left.\left\langle C_{x}\right\rangle\right|_{\beta}$, at space-time location $(x, t)$ for a given interface position, $\beta$, satisfies the following equation:

$$
\begin{gathered}
\frac{\left.\partial\left\langle C_{x}\right\rangle\right|_{\beta}}{\partial t}+\frac{\left.\partial\left\langle C_{x}\right\rangle\right|_{\beta}}{\partial x}-\frac{\partial}{\partial x}\left(\left[H(\beta-x)\left(\left\langle\alpha_{L_{1}}\right\rangle \frac{\left.\partial\left\langle C_{x}\right\rangle\right|_{\beta}}{\partial x}+\frac{\left.\partial\left\langle\alpha^{\prime}{ }_{L_{1}} C_{x}\right\rangle\right|_{\beta}}{\partial x}\right)\right.\right. \\
\left.\left.+H(x-\beta)\left(\left\langle\alpha_{L_{2}}\right\rangle \frac{\left.\partial\left\langle C_{x}\right\rangle\right|_{\beta}}{\partial x}+\frac{\left.\partial\left\langle{\alpha^{\prime}}_{L_{2}} C_{x}\right\rangle\right|_{\beta}}{\partial x}\right)\right]\right)=0
\end{gathered}
$$

subject to the initial and boundary conditions:

$$
\begin{array}{cc}
\left.\left\langle C_{x}\right\rangle\right|_{\beta}=\delta(x) \quad \text { for } 0 \leq x \leq 1, & t=0 \\
\lim _{x \rightarrow 0^{+}}\left\langle\alpha_{L_{1}}\right\rangle \frac{\left.\partial\left\langle C_{x}\right\rangle\right|_{\beta}}{\partial x}=0, \quad \frac{\left.\partial\left\langle C_{x}\right\rangle\right|_{\beta}}{\partial x}=0 \text { for } x=1, & \forall t>0
\end{array}
$$

We evaluate (11) and (12) recursively, by expanding all (statistical) moments appearing therein as

$\left.\langle\psi\rangle\right|_{\beta}=\left.\sum_{n=0}^{\infty}\langle\psi\rangle\right|_{\beta} ^{[n]}$

Here, $\left.\langle\psi\rangle\right|_{\beta} ^{[n]} \propto\left\langle\left(\alpha^{\prime}{ }_{L_{1}}\right)^{m}\right\rangle\left\langle\left(\alpha^{\prime}{ }_{L_{2}}\right)^{j}\right\rangle$ with $m+j=n$. Expansion (13) rests on the idea that each material is associated with mild values of local dispersivity variance, which is consistent with the composite medium scheme we are employing. In the following, we limit our recursive approximation to second order, i.e., $n=2$, and approximate $\left.\langle\psi\rangle\right|_{\beta} \approx$ $\left.\langle\psi\rangle\right|_{\beta} ^{[0]}+\left.\langle\psi\rangle\right|_{\beta} ^{[2]}$ (note that $\left.\langle\psi\rangle\right|_{\beta} ^{[1]}$ vanishes).

The zero- and second- order approximations of the conditional mean concentration, $\left.\left\langle C_{x}\right\rangle\right|_{\beta} ^{[k]}$, are given by

$$
\begin{aligned}
& \frac{\left.\partial\left\langle C_{x}\right\rangle\right|_{\beta} ^{[k]}}{\partial t}+\frac{\left.\partial\left\langle C_{x}\right\rangle\right|_{\beta} ^{[k]}}{\partial x}-\frac{\partial}{\partial x}\left[\left(H(\beta-x)\left\langle\alpha_{L_{1}}\right\rangle+H(x-\beta)\left\langle\alpha_{L_{2}}\right\rangle\right) \frac{\left.\partial\left\langle C_{x}\right\rangle\right|_{\beta} ^{[k]}}{\partial x}\right]= \\
& =-\frac{\partial}{\partial x}\left(H(\beta-x) \frac{\left.\partial\left\langle\alpha^{\prime}{ }_{L_{1}} C_{x}\right\rangle\right|_{\beta} ^{[k]}}{\partial x}+H(x-\beta) \frac{\left.\partial\left\langle\alpha^{\prime}{ }_{L_{2}} C_{x}\right\rangle\right|_{\beta} ^{[k]}}{\partial x}\right) k=0,2
\end{aligned}
$$

subject to the initial and boundary conditions

$$
\begin{aligned}
& \left.\left\langle C_{x}\right\rangle\right|_{\beta} ^{[k]}=\delta(x)\left[1-\frac{k}{2}\right] \quad \text { for } 0 \leq x \leq 1, \\
& \lim _{x \rightarrow 0^{+}}\left\langle\alpha_{L_{1}}\right\rangle \frac{\left.\partial\left\langle C_{x}\right\rangle\right|_{\beta} ^{[k]}}{\partial x}=0, \frac{\left.\partial\left\langle C_{x}\right\rangle\right|_{\beta} ^{[k]}}{\partial x}=0 \quad \text { for } x=1, \quad \forall t>0 \\
& t=0
\end{aligned}
$$


An expression for the second order (i.e., $k=2$ ) cross-covariance $\left.\left\langle\alpha^{\prime}{ }_{L_{i}} C_{x}\right\rangle\right|_{\beta} ^{[2]}$ (with $i=1,2$ ) is derived in Appendix A, the corresponding zero-order component being equal to zero. We note that, according to (14), both $\left.\left\langle C_{x}\right\rangle\right|_{\beta} ^{[0]}$ and $\left.\left\langle C_{x}\right\rangle\right|_{\beta} ^{[2]}$ satisfy a classical ADE whereas the source term (i) is null for $k=0$ and (ii) is a function of $\sigma_{\alpha_{L_{i}}}^{2}$ (through (A.3)) for $k=2$. In other words, $\left.\left\langle C_{x}\right\rangle\right|_{\beta} ^{[2]}$ accounts for the influence of our lack of knowledge about local dispersivity values on the expected value of the concentration field, given the location of materials' interface. Note that the term $\left.\partial\left\langle\alpha^{\prime}{ }_{L_{i}} C_{x}\right\rangle\right|_{\beta} ^{[2]} / \partial x$ in (14) is analogous to the dispersive flux appearing in Morales et al. (2006a,b), and Neuman (1993), where the MEs are formulated considering a solute transport problem characterized by a homogeneous deterministic local dispersion coefficient while there is a lack of knowledge in the hydraulic conductivity field. Given that $\left.\left\langle C_{x}\right\rangle\right|_{\beta} ^{[0]+[2]}$ is intended as our best prediction of the actual (unknown) $C_{x}$, the term $\left.\partial\left\langle\alpha^{\prime}{ }_{L_{i}} C_{x}\right\rangle\right|_{\beta} ^{[2]} / \partial x$ reflects a loss of information rather than a physical dispersive process (which in our set-up is controlled by the actual values of $\alpha_{L_{i}}$. Clearly, $\left.\left\langle C_{x}\right\rangle\right|_{\beta} ^{[2]}$ vanishes if the dispersive properties of the two materials are deterministically known, i.e., if $\sigma_{\alpha_{L_{i}}}^{2}=0$, and $C_{x}$ coincides with $\left.\left\langle C_{x}\right\rangle\right|_{\beta} ^{[0]}$.

The second-order approximation of the cross-covariance $\left.\left\langle C^{\prime}{ }_{\xi} C_{x}{ }_{x}\right\rangle\right|_{\beta}$ (see Appendix B) is given by

$$
\begin{gathered}
\frac{\left.\partial\left\langle C^{\prime}{ }_{\xi} C^{\prime}{ }_{x}\right\rangle\right|_{\beta} ^{[2]}}{\partial t}+\frac{\left.\partial\left\langle C^{\prime}{ }_{\xi} C^{\prime}{ }_{x}\right\rangle\right|_{\beta} ^{[2]}}{\partial x}-\frac{\partial}{\partial x}\left[\left(H(\beta-x)\left\langle\alpha_{L_{1}}\right\rangle+H(x-\beta)\left\langle\alpha_{L_{2}}\right\rangle\right) \frac{\partial\left\langle\overline{\left.\left\langle C^{\prime}{ }_{\xi} C^{\prime}{ }_{x}\right\rangle\right|_{\beta} ^{[2]}}\right.}{\partial x}\right] \\
=\frac{\partial}{\partial x}\left[\left(\left.H(\beta-x)\left\langle\alpha^{\prime}{ }_{L_{1}} C^{\prime}{ }_{\xi}\right\rangle\right|_{\beta} ^{[2]}+\left.H(x-\beta)\left\langle\alpha^{\prime}{ }_{L_{2}} C^{\prime}{ }_{\xi}\right\rangle\right|_{\beta} ^{[2]}\right) \frac{\left.\partial\left\langle C_{x}\right\rangle\right|_{\beta} ^{[0]}}{\partial x}\right]
\end{gathered}
$$

subject to the initial and boundary conditions

$$
\begin{aligned}
& \left.\left\langle C^{\prime}{ }_{\xi} C^{\prime}{ }_{x}\right\rangle\right|_{\beta} ^{[2]}=0 \quad \text { for } 0 \leq x \leq 1, \quad t=0 \\
& \lim _{x \rightarrow 0^{+}}\left\langle\alpha_{L_{1}}\right\rangle \frac{\left.\partial\left\langle C^{\prime}{ }_{\xi} C^{\prime}{ }_{x}\right\rangle\right|_{\beta} ^{[2]}}{\partial x}+\left.\left\langle\alpha^{\prime}{ }_{L_{1}} C^{\prime}{ }_{\xi}\right\rangle\right|_{\beta} ^{[2]} \frac{\left.\partial\left\langle C_{x}\right\rangle\right|_{\beta} ^{[0]}}{\partial x}=0, \\
& \frac{\left.\partial\left\langle C^{\prime}{ }_{\xi} C^{\prime}{ }_{x}\right\rangle\right|_{\beta} ^{[2]}}{\partial x}=0 \text { for } x=1, \quad \forall t>0
\end{aligned}
$$

where $(\xi, \tau)$ indicate space and time coordinates. The conditional concentration variance at space-time location $(x, t)$ can then be obtained through (16)-(17) as

$\sigma_{\left.C_{x}\right|_{\beta}}^{2}=\left.\lim _{\substack{\xi \rightarrow x \\ \tau \rightarrow t}}\left\langle C^{\prime}{ }_{\xi} C^{\prime}{ }_{x}\right\rangle\right|_{\beta} ^{[2]}$

Note that a source term which is a function of $\sigma_{\alpha_{L_{i}}}^{2}$ (through A.3) appears on the right hand side of (16). This term vanishes in the presence of deterministically known material dispersivities, thus yielding $\sigma_{\left.C_{x}\right|_{\beta}}^{2}=$ 0 , given the homogenous boundary conditions in (17).

\subsubsection{Moment equations for solute concentration with uncertain dispersivities and geomaterial interface location}

Removing conditioning on $\beta$ from the (ensemble) moments considered in Section 2.2.1 yields global (or unconditional) moments of quantities of interest. Such (ensemble) moments imbue the contribution of uncertainties in the location of the interface as well as in the local dispersivity values.

Following (9), the (unconditional) mean concentration, $\left\langle C_{x}\right\rangle$, can be evaluated as

$\left\langle C_{x}\right\rangle=\left.\int_{\Gamma_{\beta}}\left\langle C_{x}\right\rangle\right|_{\beta} p(\beta) d \beta$

Evaluating concentration variance $\sigma_{C_{x}}^{2}$ at a given space-time location entails writing concentration fluctuations as

$C_{x}^{\prime}=\left.C^{\prime}{ }_{x}\right|_{\beta}+\left.\overline{C_{x}}\right|_{\beta}$ where we decompose the concentration fluctuation according to the contribution of two terms: (a) $\left.C_{x}^{\prime}\right|_{\beta}=\left.C_{x}\right|_{\beta}-\left.\left\langle C_{x}\right\rangle\right|_{\beta}$, representing the concentration fluctuation for a given interface position, and $\left.(b) \overline{C_{x}}\right|_{\beta}=$ $\left.\left\langle C_{x}\right\rangle\right|_{\beta}-\left\langle C_{x}\right\rangle$, representing the difference between the conditional and the unconditional mean concentration. One can then write the following equation for $\sigma_{C_{x}}^{2}$

$$
\begin{aligned}
\sigma_{C_{x}}^{2} & =\iiint_{\Gamma_{\beta}, \Gamma_{\alpha_{L}}, \Gamma_{\alpha_{L_{2}}}}\left(\left.C^{\prime}{ }_{x}\right|_{\beta}+\left.\bar{C}_{x}\right|_{\beta}\right)^{2} p(\beta) p\left(\alpha_{L_{1}}\right) p\left(\alpha_{L_{2}}\right) d \beta d \alpha_{L_{1}} d \alpha_{L_{2}} \\
& =\int_{\Gamma_{\beta}} \sigma_{\left.C_{x}\right|_{\beta}}^{2} p(\beta) d \beta+\left.\int_{\Gamma_{\beta}} \bar{C}_{x}\right|_{\beta} ^{2} p(\beta) d \beta=\left\langle\sigma_{\left.C_{x}\right|_{\beta}}^{2}\right\rangle+\left\langle\left.\bar{C}_{x}\right|_{\beta} ^{2}\right\rangle
\end{aligned}
$$

Eq. (21) clearly highlights that the uncertainty (as quantified by the variance) of the solute concentration is due to the sum of two terms. The first one, $\left\langle\sigma_{\left.C_{x}\right|_{\beta}}^{2}\right\rangle$, is the mean value (evaluated in the space of variability of $\beta$ ) of the variance of the concentration associated with the uncertainty of the local dispersivities. The second term, $\left\langle\left.\overline{C_{x}}\right|_{\beta}{ }^{2}\right\rangle$, represents the contribution to $\sigma_{C_{x}}^{2}$ due to the variability of the mean concentration arising from conditioning on $\beta$. Clearly, $\sigma_{\left.C_{x}\right|_{\beta}}^{2}$ and $\left\langle\left.\overline{C_{x}}\right|_{\beta} ^{2}\right\rangle$ vanish if one deterministically knows the local dispersivity values or the position of the interface between the two porous domains, respectively.

\subsection{Numerical implementation}

Solution of (14)-(21) requires accurate numerical methods to limit numerical dispersion and properly describe the behavior of the target statistical moments. Following Cockburn and Shu (1998) and Di Pietro and Ern (2012), we apply the discontinuous Galerkin finite element method to the hyperbolic (advective) part of (14) and (16). Details about the adopted numerical method are illustrated in Appendix C.

We evaluate the unconditional concentration mean, i.e., $\left\langle C_{x}\right\rangle$, by solving (16)-(17) for $N_{\beta}$ locations of the interface, randomly sampled from $p(\beta)$. We then approximate (19) as

$\left\langle C_{x}\right\rangle \approx \frac{1}{N_{\beta}} \sum_{i=1}^{N_{\beta}}\left(\left.\left\langle C_{x}\right\rangle\right|_{\beta_{i}} ^{[0]}+\left.\left\langle C_{x}\right\rangle\right|_{\beta_{i}} ^{[2]}\right)$

where $\left.\left\langle C_{x}\right\rangle\right|_{\beta_{i}} ^{[0]}$ and $\left.\left\langle C_{x}\right\rangle\right|_{\beta_{i}} ^{[2]}$ are the zero- and second-order components of the expected value of concentration conditional to $\beta=\beta_{i}$.

The (unconditional) concentration variance is evaluated approximating (21) as

$\sigma_{C_{x}}^{2}=\frac{1}{N_{\beta}} \sum_{i=1}^{N_{\beta}}\left\{\sigma_{\left.C_{x}\right|_{\beta_{i}} ^{2}}^{2}+\left[\left(\left.\left\langle C_{x}\right\rangle\right|_{\beta_{i}} ^{[0]}+\left.\left\langle C_{x}\right\rangle\right|_{\beta_{i}} ^{[2]}\right)-\left(\left\langle C_{x}\right\rangle^{[0]}+\left\langle C_{x}\right\rangle^{[2]}\right)\right]^{2}\right\}$

where $\sigma_{\left.C_{x}\right|_{\beta_{i}}}^{2}$ is the numerical solution of (17) and (18) conditional to a given $\beta_{i}$.

\section{Computational showcases: results and discussion}

We explore the implications of conceptualizing the porous domain as a random composite (in the sense illustrated in Section 2) where each geomaterial is characterized by random local dispersivities by way of two 
arrangements of the sub-domains forming the system: $(i)$ a coarse to fine arrangement (hereafter denoted as $\mathrm{C}-\mathrm{F}$ ), where a coarse geomaterial precedes a fine one along the direction of flow; and (ii) a fine to coarse arrangement (hereafter termed F-C). We set $\left\langle\alpha_{L_{1}}\right\rangle=\alpha_{L_{C}}=2.5 \times 10^{-2}$ and $\left\langle\alpha_{L_{2}}\right\rangle=\alpha_{L_{F}}=2.5 \times 10^{-3}$ in the C-F setting, the F-C scenario being associated with the opposite sequence of values. In both scenarios $\langle\beta\rangle=0.5, \alpha_{L_{1}}, \alpha_{L_{2}}$, and $\beta$ being modeled as independent and identically distributed random variables characterized by uniform densities. This choice is consistent with a setting where no prior information on $\alpha_{L_{i}}$ and $\beta$ is available, alternative choices about the distribution of $\alpha_{L_{i}}$ and $\beta$ being fully compatible with the developed approach. For the sake of our analyses, we consider a coefficient of variation equal to $20 \%$ for all distributions. This choice enables us (a) to analyze composite media where the (random) location of the material interface is always included in the studied domain, and $(b)$ to explore the behavior of a well demarcated system where local dispersivities of the fine and coarse media are associated with differing orders of magnitude and their probability density functions are characterized by non-overlapping supports.

The conditional moment equations are solved according to the approach illustrated in Appendix C with $N_{E}=1000$ elements (corresponding to $\Delta x=10^{-3}$ ) and a time step of $\Delta t=5^{-3}$ for a total of $N_{T}=2000$ time steps (all symbols are defined in Appendix C). These parameters provide a sufficient degree of numerical accuracy for our solution (details not shown). We find that considering $N_{\beta} \approx 170$ is sufficient to provide stable results for the moments investigated. In the following, we illustrate our key findings by focusing on three selected observation times, i.e., $t_{1}=1 / 4, t_{2}=1 / 2 ; t_{3}=3 / 4$. Our ME-based outcomes are also compared against a suite of numerical Monte Carlo (MC) results. The latter have been evaluated with the same numerical technique and space-time discretization employed to compute the conditional MEs and by relying on a collection of $1000 \mathrm{MC}$ replicates for each given location of the material interface. Note that, even as the computational costs of a standard Monte Carlo approach are easily affordable for the exemplified case, the MEs approach allows for an improved understanding of the way material dispersivities and interface location uncertainty affect the leading statistical moments of the solute concentration, as discussed in Section 2.

\subsection{Numerical results for unknown dispersivities and known interface location}

Fig. 1 depicts the dependence on $x$ of $(\mathrm{a}-\mathrm{c})$ the conditional expected value of solute concentration, i.e., $\left.\left\langle C_{x}\right\rangle\right|_{\beta=0.5}$, (d-f) the corresponding conditional concentration variance, i.e., $\sigma_{\left.C_{x}\right|_{\beta=0.5} ^{2}}^{2}$, and (g-i) the associated coefficient of variation, i.e., $\left.C V_{C_{x}}\right|_{\beta=0.5}=\sigma_{\left.C_{x}\right|_{\beta=0.5}} /\left.\left\langle C_{x}\right\rangle\right|_{\beta=0.5}$, at the three selected observation times in the (C-F) setting for $\beta=0.5$. ME(red curves) and MC- (blue curves) based results are depicted. Fig. 1a-c also depicts three randomly selected spatial profiles of $\left.C_{x}\right|_{\beta=0.5}$. Corresponding results for the $(\mathrm{F}-\mathrm{C})$ setting are illustrated in Fig. 2. In general, ME- and MC- based results display a good agreement for all cases and quantities analyzed, the highest ME-based peak value of $\sigma_{\left.C_{x}\right|_{\beta=0.5}}^{2}$ slightly underestimating its MC-based counterpart (see Figs. $1 \mathrm{~d}-\mathrm{f}$ and 2d-f).

Our results show that for a given observation time the average behavior of the solute is characterized by a higher spatial spreading of the plume in the $(\mathrm{C}-\mathrm{F})$ than in the $(\mathrm{F}-\mathrm{C})$ scenario. To explore this pattern, we consider a new dimensionless time, $\tilde{t}$, obtained by rescaling the (dimensional) observation time $t^{*}$ with respect to a characteristic mean dispersive time, i.e., $\tilde{t}=t^{*} u^{*}\left\langle\alpha_{L_{i}}^{*}\right\rangle / L^{* 2}$. It then follows that such a dimensionless time corresponds to $\widetilde{t}_{C}=t \alpha_{L_{C}}$ or $\widetilde{t}_{F}=t \alpha_{L_{F}}$, for the coarse or the fine geomaterial, respectively. Note that $\widetilde{t}_{F} / \widetilde{t}_{C}=\alpha_{L_{F}} / \alpha_{L_{C}}=0.1$ in our test case, i.e., the local dispersive mechanism in the fine geomaterial acts slower than in the coarse one. This latter observation implies that the solute migrating across the fine geomaterial should travel (on average) ten times the corresponding distance traversed within the coarse geomaterial to attain the same level of spreading, i.e., to experience the same total amount of local dispersive length scales. According to this conceptual picture, we note that the plume spreading at advective time $t_{1}$ (corresponding to an observation time where the influence of the presence of the second material on the average solute concentration can be neglected) is at a more advanced stage for the (C-F) than for the (F-C) arrangement, thus explaining why $\left.\left\langle C_{x}\right\rangle\right|_{\beta=0.5}$ in Fig. 1a appears to be more spread across space than in Fig. 2a. When the solute experiences the effects of two geomaterials (e.g., at advective times $t_{2}$ and $t_{3}$ ), the plume spreading behavior is controlled by the amount of characteristic local dispersive lengths ( $\alpha_{L_{C}}$ and $\alpha_{L_{F}}$ for the coarse and fine materials, respectively) traveled by the solute in both geomaterials.

The shape of the mean concentration profiles (and that of the randomly chosen $C_{x}$ ) shows a distinctive pattern in the proximity of the interface, where the spatial derivate of $\left.\left\langle C_{x}\right\rangle\right|_{\beta=0.5}$ is lower in the coarse than in the fine material (see Figs. $1 \mathrm{a}-\mathrm{c}$ and $2 \mathrm{a}-\mathrm{c}$ ). This behavior is a consequence of the continuity of solute flux across the interface, according to which low concentration gradients are associated with high values of local dispersivity and vice-versa. The interface also affects the location of the peaks of $\left.\left\langle C_{x}\right\rangle\right|_{\beta=0.5}$. For example, at time $t_{2}$, the peak of the concentration profile in a homogeneous domain would occur at $x=0.5$, while in our settings it always resides in the portion of the domain occupied by the fine geomaterial (see Figs. $1 \mathrm{~b}$ and $2 \mathrm{~b}$ ). Note that the analyses illustrated so far highlight that the plume-scale spreading behavior (as manifested in each $C_{x}$ realization and in the resulting $\left.\left\langle C_{x}\right\rangle\right|_{\beta=0.5}$ ) is non-Fickian due to heterogeneity in the local dispersivities and to the presence of the materials' interface.

The conditional concentration variance, $\sigma_{\left.C_{x}\right|_{\beta=0.5}}^{2}$, displays three peaks and two local minima. This finding is consistent with the physical nature of the process analyzed. In each realization the solute is dispersed from the center of mass of the advancing plume (the position of which is dictated by the deterministic velocity $u^{*}$ ) towards the tails with a strength governed by the uncertain material dispersivities. Considering the three random realizations depicted in Figs. 1a-c and 2a-c, one can see that solute concentrations profiles corresponding to peak values of $\left.C_{x}\right|_{\beta=0.5}$ lower than $\left.\left\langle C_{x}\right\rangle\right|_{\beta=0.5}$ exhibit tails heavier than those of $\left.\left\langle C_{x}\right\rangle\right|_{\beta=0.5}$, the opposite being observed when the peak of $\left.C_{x}\right|_{\beta=0.5}$ is larger than the one displayed by $\left.\left\langle C_{x}\right\rangle\right|_{\beta=0.5}$. This mechanism causes the occurrence of the two observed local minima (and three peaks). We further note that the highest peak of $\sigma_{\left.C_{x}\right|_{\beta=0.5} ^{2}}$ is located in the proximity of the peak of $\left.\left\langle C_{x}\right\rangle\right|_{\beta=0.5}$.

Comparison of Figs. $2 \mathrm{~d}-\mathrm{f}$ and $1 \mathrm{~d}-\mathrm{f}$ shows that $\sigma_{\left.C_{x}\right|_{\beta=0.5}}^{2}$ attains larger values in the (F-C) than in the (C-F) scenario. This result could appear as counterintuitive, at least fort $t_{1}$ (i.e., when solely the first geomaterial dispersivity dictates the spreading behavior of the plume), because the local dispersivity variance of the coarse geomaterial is larger than that of the fine unit and one might expect larger values of $\sigma_{\left.C_{x}\right|_{\beta=0.5}}^{2}$ in the (C-F) than in the $(\mathrm{F}-\mathrm{C})$ setting. This apparent inconsistency is reconciled by recalling that the local dispersive mechanism acts faster in the coarse than in the fine geomaterial. Therefore, concentration profiles (at a given time) in the coarse geomaterial tend to display more enhanced spreading and dilution than in the fine medium. Thus, differences among the $\left.C_{x}\right|_{\beta=0.5}$ profiles (as quantified by the variance $\sigma_{\left.C_{x}\right|_{\beta=0.5}}^{2}$ ) across the collection of MC realizations at a given time are significantly larger in the fine than in the coarse system. This observation is further supported by noticing that the drop of $\sigma_{\left.C_{x}\right|_{\beta=0.5}}^{2}$ observed comparing $t_{2}$ and $t_{3}$ is smaller than that taking place between $t_{1}$ and $t_{2}$ in (C-F), i.e., it appears that the spreading mechanism tends to diminish its strength due to the presence of the fine geomaterial. The opposite holds for the (F-C) arrangement.

The (conditional) variance $\sigma_{\left.C_{x}\right|_{\beta=0.5}}^{2}$ is initially zero, due to the deterministic conditions imposed. The injected pulse then migrates according 

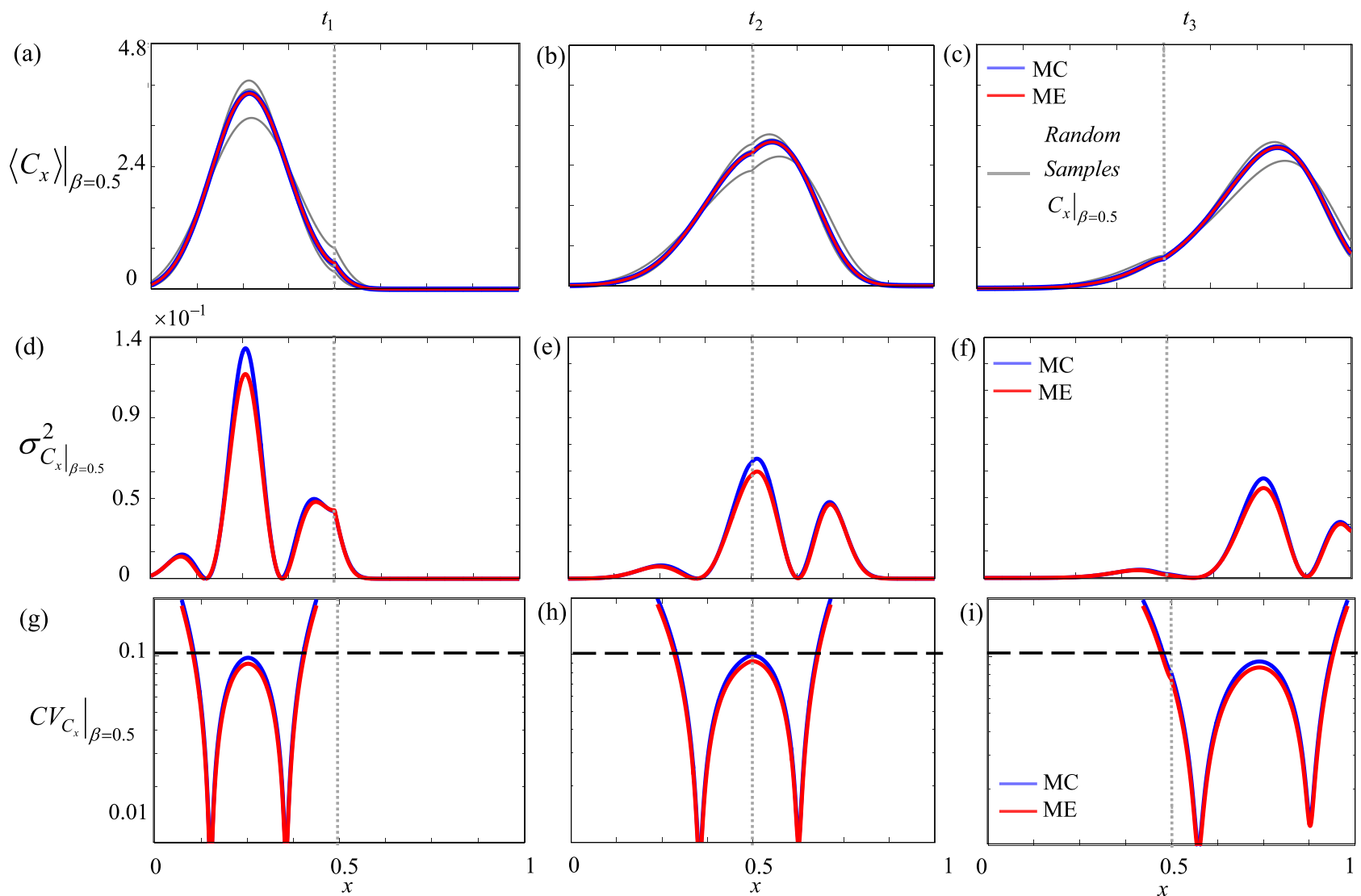

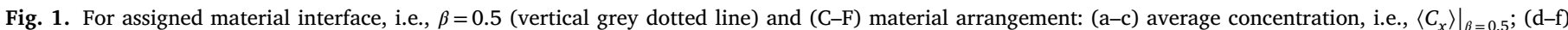

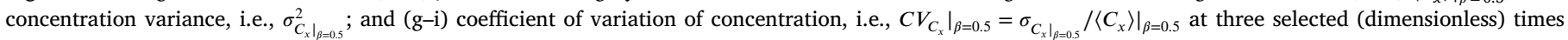

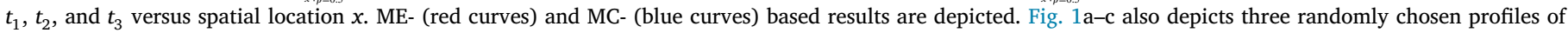
$\left.C_{x}\right|_{\beta=0.5}$ (grey curves). (For interpretation of the references to color in this figure legend, the reader is referred to the web version of this article.)

to a deterministic advective velocity and spreads according to an uncertain dispersivity value, the latter governing the strength of $\sigma_{\left.C_{x}\right|_{\beta=0.5}}^{2}$. As time progresses, the effect of local dispersion leads to have more spread in the solute plumes, i.e., the peak value of $\left.\left\langle C_{x}\right\rangle\right|_{\beta=0.5}$ drops and $\left.\left\langle C_{x}\right\rangle\right|_{\beta=0.5}$ encompasses increased portions of the domain. Since there is a constant solute mass in each realization, the differences between the concentration profiles across the collection of MC realizations tend to decrease as time increases. This is also related to the observation that the maximum value of $\sigma_{\left.C_{x}\right|_{\beta=0.5}}^{2}$ tends to decrease and the values of $\sigma_{\left.C_{x}\right|_{\beta=0.5}}^{2}$ to spread over space with time.

The insets in Fig. 2c and f respectively juxtapose $\left.\left\langle C_{x}\right\rangle\right|_{\beta=0.5}$ and $\sigma_{\left.C_{x}\right|_{\beta=0.5}}^{2}$ evaluated through our MEs for the (C-F) (solid curve) and the (F-C) (dashed curve) arrangements. The (C-F) and (F-C) solutions coincide at a spatial location twice the value of $\beta$ (i.e., at the outlet $x=1$ for $\beta=0.5$ ). This follows from the observation that at this location the solute has experienced (on average) the same total amount of dispersive length scales (in terms of $\alpha_{L_{C}}$ and $\alpha_{L_{F}}$ ) in both geomaterial arrangements. The same observation holds for any fixed value of $\beta$.

The analysis of the coefficient of variation $\left.C V_{C_{x}}\right|_{\beta=0.5}$ reveals that as far as the solute plume spreads solely within one geomaterial, e.g., at time $t_{1}$ (Figs. $1 \mathrm{~g}$ and $2 \mathrm{~g}$ ), the relative discrepancy between profiles of $\left.C_{x}\right|_{\beta=0.5}$ across the collection of realizations and their (ensemble) mean shows some similarities in both (C-F) and (F-C) settings. Some details of this aspect are elucidated by the inset of Fig. $2 \mathrm{~g}$ that juxtaposes $\left.C V_{C_{\gamma}}\right|_{\beta=0.5}$ evaluated through our MEs for the (C-F) (dashed curve) and the (F-C) (solid curve) arrangements at time $t_{1}$. Here, one can note that the maximum value of $\left.C V_{C_{x}}\right|_{\beta=0.5}$ attains the same value and occurs at the same spatial location (i.e., at the advective dimensionless distance $x=t_{1}$ ) for both settings. Our results also show that $\left.C V_{C_{x}}\right|_{\beta=0.5}$ versus $\tilde{x}=x-t$ at $t=t_{1}$ for $(\mathrm{F}-\mathrm{C})$ coincides with its counterpart evaluated at $t_{1} / 10$ for (C-F).

At later times, e.g., $t_{3}$, when the solute has traveled through both geomaterials, $\left.C V_{C_{x}}\right|_{\beta=0.5}$ decreases for both geomaterial arrangements (see Figs. 1i, and $2 \mathrm{i}$ ). The latter result is consistent with the observation that the joint action of diverse combinations of local dispersivity values characterizing the two units can lead to the same value of concentration at a given space-time location (see also our previous comments about the system behavior at $x=1$ ). In other words, our results reveal that the relative discrepancy between the $\left.C_{x}\right|_{\beta=0.5}$ spatial profiles that one can observe across a collection of Monte Carlo realizations tends to decrease as a consequence of the heterogeneity of the porous media dispersive properties as quantified at a given spatial scale.

\subsection{Numerical results for unknown dispersivities and unknown interface location}

We now analyze the leading ensemble moments of concentration by considering the joint effects of uncertainty of local dispersivities and geomaterials' interface. Fig. 3a-c depict the mean concentration, $\left\langle C_{x}\right\rangle$, versus $x$ in the (C-F) setting at the three selected observation times. Results obtained through MEs (red curves) and MC (blue curves) are displayed. For comparison purposes, Fig. 3a-c include depictions of the conditional mean concentrations $\left.\left\langle C_{x}\right\rangle\right|_{\beta=\beta_{\min }}$ (black curves), $\left.\langle C\rangle\right|_{\beta=0.5}$ (green curves) and $\left.\left\langle C_{x}\right\rangle\right|_{\beta=\beta_{\max }}$ (purple curves) obtained by setting the interface at the locations corresponding to the minimum $\left(\beta_{\min }\right)$, mean, and maximum ( $\left.\beta_{\max }\right)$ value characterizing the distribution of $\beta$. Fig. $3 \mathrm{~d}-$ 

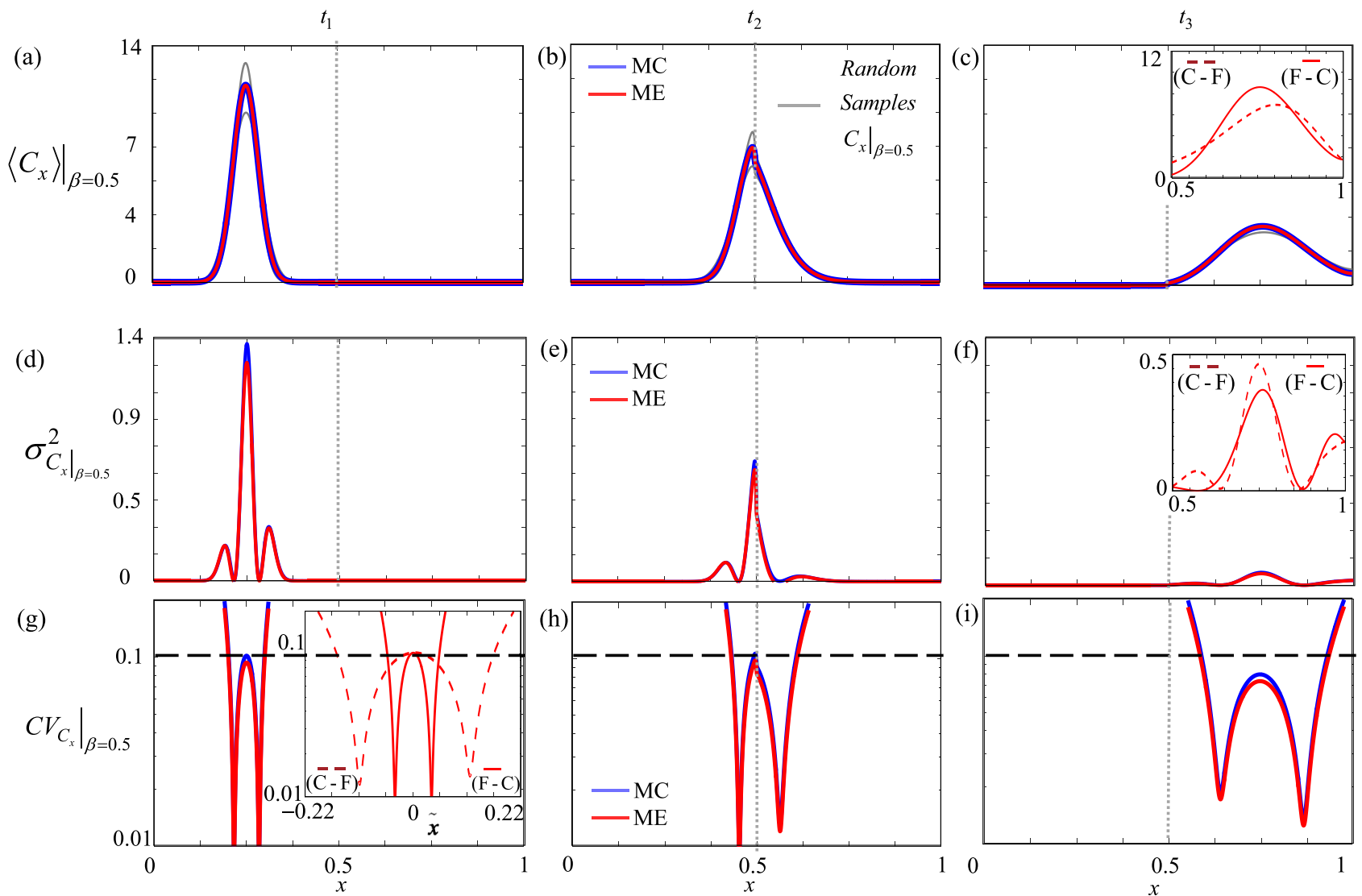

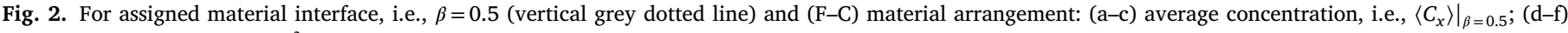

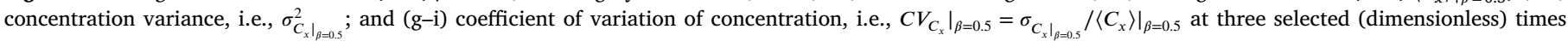

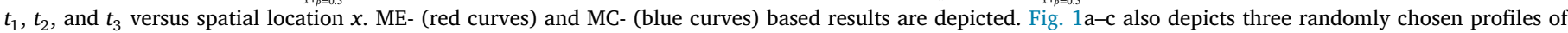

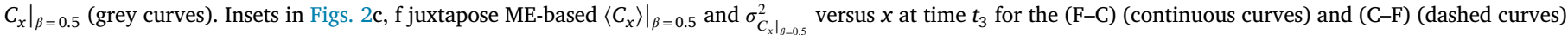

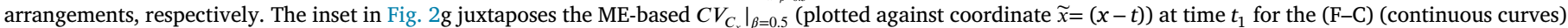

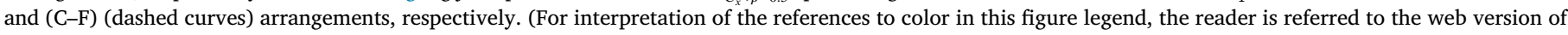
this article.)
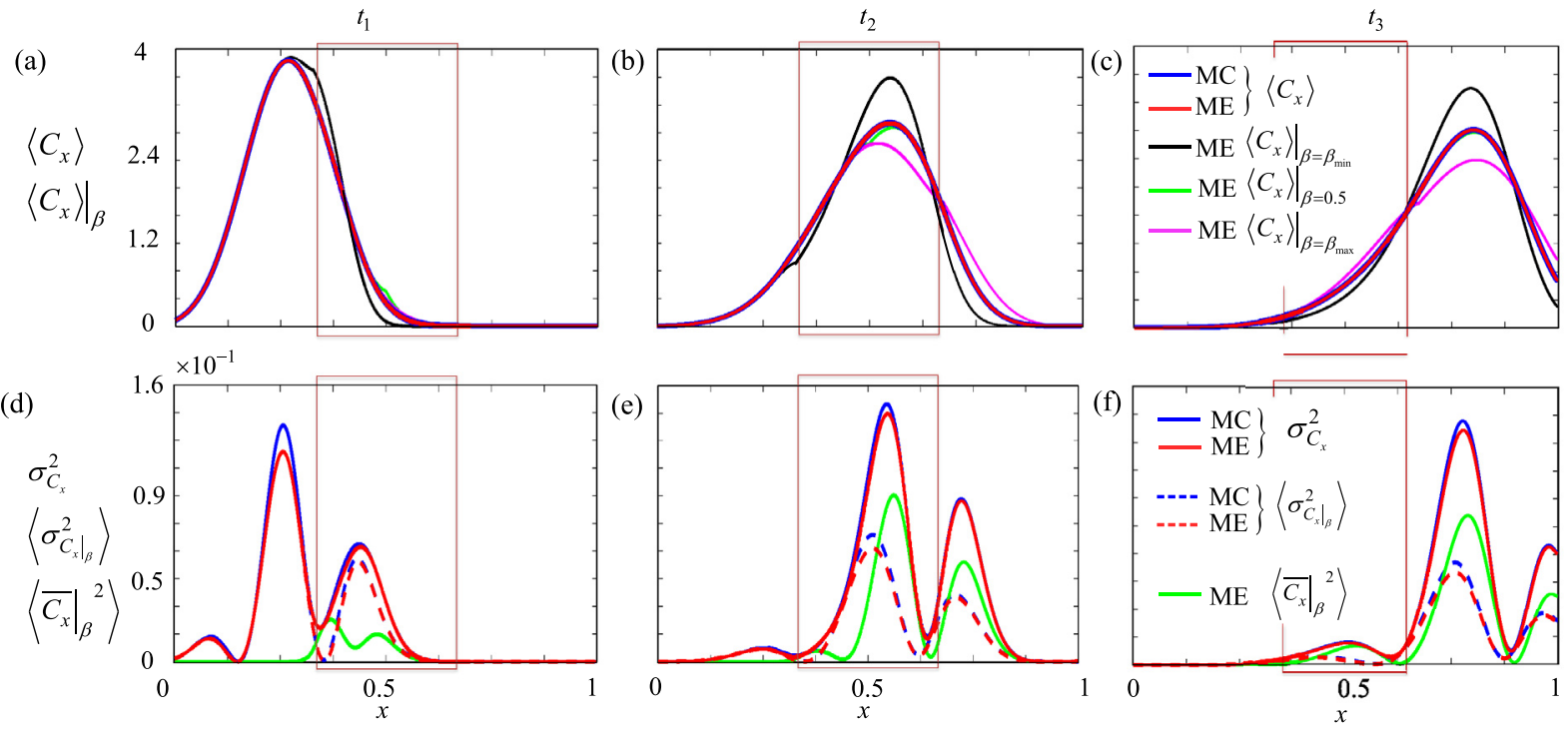

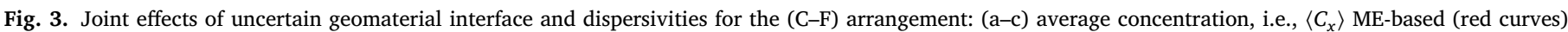

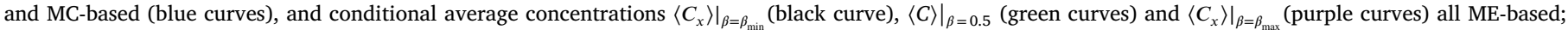

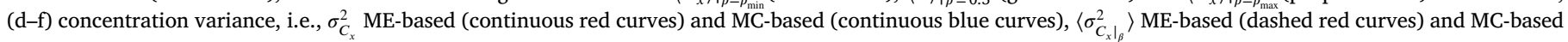

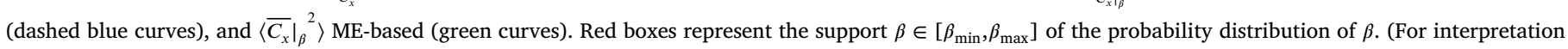
of the references to color in this figure legend, the reader is referred to the web version of this article.) 

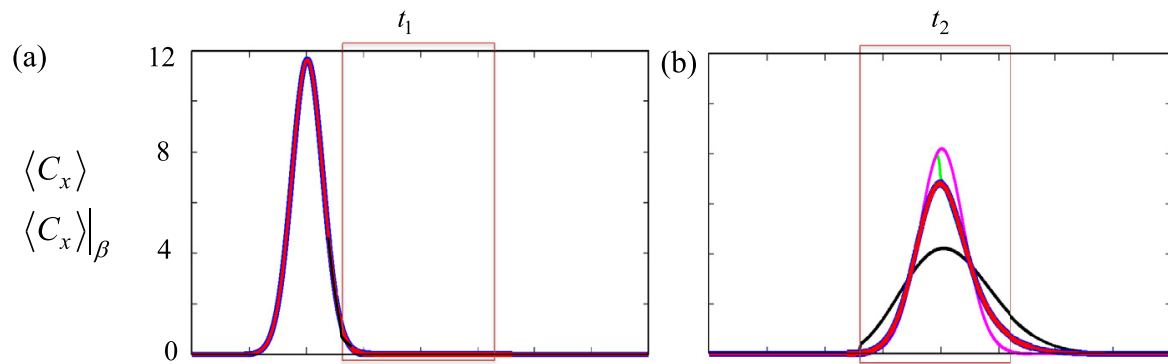

(d)

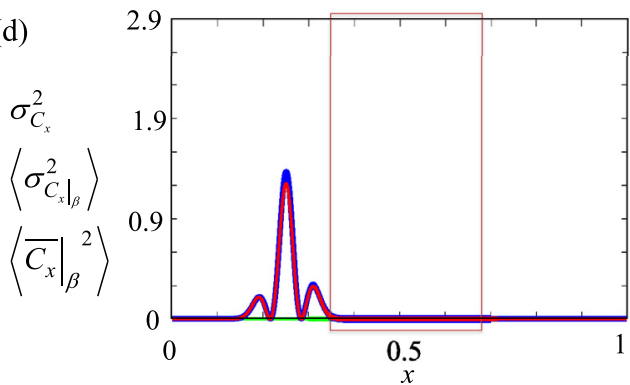

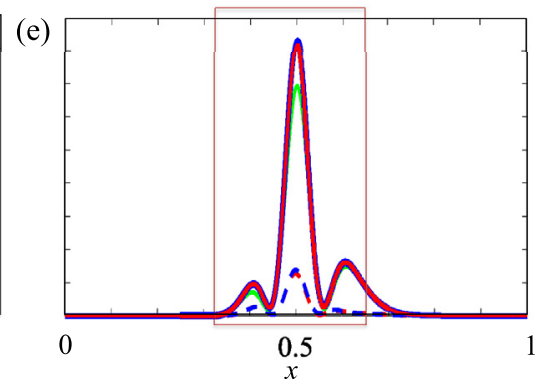

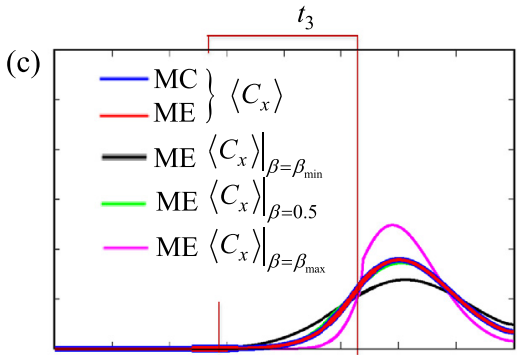

(f)

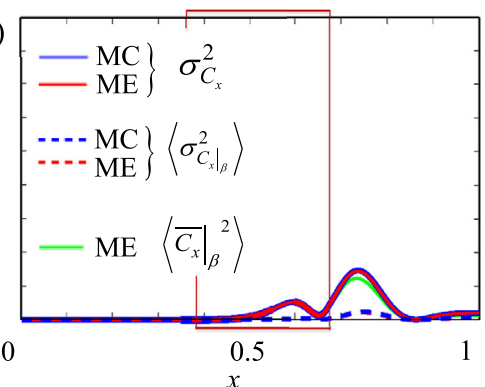

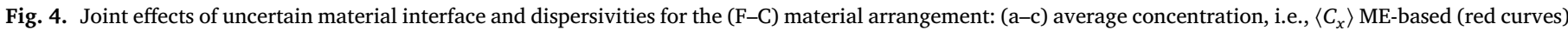

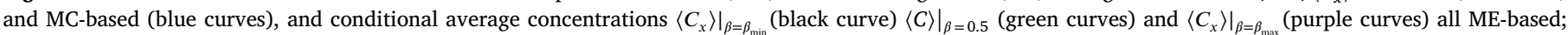

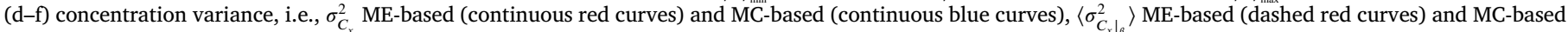

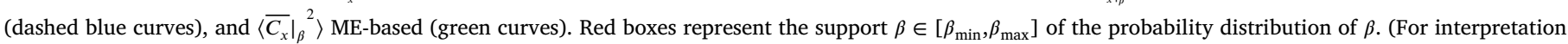
of the references to color in this figure legend, the reader is referred to the web version of this article.)

f depict concentration variance $\sigma_{C_{x}}^{2}$ computed via MEs (continuous red curves) and MC (continuous blue curves). For completeness, the secondorder (ME-based) approximation of the two terms in (23) contributing to $\sigma_{C_{x}}^{2}$, i.e., $\left\langle\sigma_{\left.C_{x}\right|_{\beta}}^{2}\right\rangle$ and $\left\langle\left.\overline{C_{x}}\right|_{\beta}{ }^{2}\right\rangle$, together with the MC-based evaluation of $\left\langle\sigma_{\left.C_{x}\right|_{\beta}}^{2}\right\rangle$ are also depicted. The MC computed $\left\langle\left.\overline{C_{x}}\right|_{\beta}{ }^{2}\right\rangle$ (not reported) practically coincides with its ME counterpart, the latter depending only on the second-order conditional mean concentration field (see (23)). We also note that the ME-based $\left\langle\sigma_{\left.C_{x}\right|_{\beta}}^{2}\right\rangle$ is in good agreement with its MC counterpart, only slightly underestimating the MC-based peak value. Corresponding curves for the (F-C) arrangements are shown in Fig. 4.

Ensemble average spatial profiles $\left\langle C_{x}\right\rangle$ do not display discontinuities of the kind shown in Figs. 1 and 2 for the conditional $\left.\left\langle C_{x}\right\rangle\right|_{\beta}$. This is related to the observation that $\left.\left\langle C_{x}\right\rangle\right|_{\beta}$ displays a discontinuity in its derivative only close to $\beta$. Thus, averaging $\left.\left\langle C_{x}\right\rangle\right|_{\beta}$ over all possible realizations of $\beta$ yields a regular $\left\langle C_{x}\right\rangle$ profile. This finding is consistent with the smoothing of the profiles of averaged heads due to randomness of the internal boundary between two media of distinct conductivities observed by Winter and Tartakovsky (2000) and Guadagnini et al. (2003). We further note that $\left\langle C_{x}\right\rangle$ does not coincide (in general) with $\left.\left\langle C_{x}\right\rangle\right|_{\beta=0.5}$, i.e., the expected value of the solute concentration cannot be accurately evaluated simply by conditioning on the mean value of the material interface position and is affected by the uncertainty of the interface location. Neglecting the latter source of uncertainty would result in a significant error in the prediction of both the peak and the spread of the mean concentration profile.

Figs. $3 \mathrm{~d}-\mathrm{f}$ and $4 \mathrm{~d}-\mathrm{f}$ show that, even as $\left\langle\sigma_{\left.C_{x}\right|_{\beta}}^{2}\right\rangle$ decreases with time (see also our previous discussion about $\sigma_{\left.C_{x}\right|_{\beta}}^{2}$ in Section 3.1), the total variance $\sigma_{C_{x}}^{2}$ increases from $t_{1}$ to $t_{2}$. This is related to our lack of knowledge about the interface position, as quantified by the contribution of $\left\langle\left.\overline{C_{x}}\right|_{\beta}{ }^{2}\right\rangle$ in (21). At short time (e.g., $t_{1}$ ), the presence of the second material has a low (see Fig. 3d) or negligible (see Fig. 4b) impact on $\sigma_{C_{x}}^{2}$. As time progresses, the second material contributes to the variability of the spreading behavior of the traveling solute, leading to increased val- ues of $\sigma_{C_{x}}^{2}$. The same line of reasoning explains the observed drop of the contribution due to $\left\langle\left.\overline{C_{x}}\right|_{\beta}{ }^{2}\right\rangle$ at time $t_{3}$. Note that the typical three-peak behavior previously observed for $\sigma_{\left.C_{x}\right|_{\beta=0.5} ^{2}}$ is also a distinctive signature of $\left\langle\left.\overline{C_{x}}\right|_{\beta}{ }^{2}\right\rangle$. The latter finding is rooted in the same mechanisms dictating the spatial pattern of $\sigma_{\left.C_{x}\right|_{\beta=0.5}}^{2}$. This is clearly seen by recalling (21) and noting that the term $\left\langle\left.\overline{C_{x}}\right|_{\beta}{ }^{2}\right\rangle$ quantifies the variability of $\left.\left\langle C_{x}\right\rangle\right|_{\beta}$ with respect to $\left\langle C_{x}\right\rangle$. When the effect of the coarse material is dominant (e.g., consider $\left.\left\langle C_{x}\right\rangle\right|_{\beta_{\max }}$ for (C-F)), $\left.\left\langle C_{x}\right\rangle\right|_{\beta}$ is lower than $\left\langle C_{x}\right\rangle$ at the plume center and exhibits higher tails than does $\left\langle C_{x}\right\rangle$, the opposite being observed when the effect of the fine material is dominant (e.g., consider $\left.\left\langle C_{x}\right\rangle\right|_{\beta_{\min }}$ for (C-F)). It then follows that the degree of spreading around its center of mass (whose location is dictated by the deterministic fluid velocity) of the profile of $\left.\left\langle C_{x}\right\rangle\right|_{\beta}$ at a given time markedly depends on the location of $\beta$.

A close inspection of Figs. 3 and 4 reveals that values of $\left\langle C_{x}\right\rangle,\left\langle\sigma_{\left.C_{x}\right|_{\beta}}^{2}\right\rangle$ and $\left\langle\left.\overline{C_{x}}\right|_{\beta}{ }^{2}\right\rangle$ calculated for the (C-F) and (F-C) settings coincide at a location equal to twice the value of $\langle\beta\rangle$ (details not shown). This finding is in agreement with our previous observation about the dependency of the conditional moments on the average dispersive length scales traveled by the solute. In other words, for the setting here analyzed the solution of the mean and variance of concentrations is insensitive to the material arrangement at a spatial location equal to twice the value of $\langle\beta\rangle$.

\section{Conclusions}

We consider solute transport taking place in a composite porous medium in the presence of uncertainty about (i) the position of the interface between two distinct geomaterials, and (ii) the dispersivity value within each geomaterial (i.e., the local dispersivity). We derive and solve Moment Equations (MEs) for the first two (statistical) moments (i.e., mean and variance) of solute concentration. We analyze settings corresponding to two arrangements of the sub-domains forming the system: a 
coarse to fine (denoted as C-F) and a fine to coarse arrangement (termed F-C). Our work leads to the following major conclusions.

1. Our MEs clearly show that it is possible to decompose the concentration variance as the sum of two distinct contributions. These are respectively associated with our lack of knowledge on the geomaterial dispersivities and on the location of the interface between the two geomaterials. Our ME-based results compare well with those obtained through a numerical Monte Carlo approach in all configurations analyzed, either in the presence of information about the geomaterial interface knowledge or when the latter is modeled as a random variable.

2. The spatial distribution of the conditional solute concentration variance exhibits a characteristic three-peak pattern with two local minima. This behavior is governed by the effect of the random local dispersivity values which dictate the strength of solute spreading around the center of mass across the ensemble of realizations. The same behavior is recovered for the contribution to the solute concentration variance associated with our lack of knowledge of the geomaterial interface.

3. In our set-up the behavior of the (ensemble) moments of solute concentrations at a given space and time coordinate is driven by the overall amount of dispersive length scales traveled (on average) by the solute. As consequence, for a given known location $(\beta)$ of the geomaterial interface, the conditional mean and variance of concentration in the (C-F) and (F-C) arrangements coincide at a location $x=2 \beta$. Similar results hold at $x=2\langle\beta\rangle$ when randomness of $\beta$ and local dispersivities are jointly considered.

4. The spatial distribution of the conditional coefficient of variation of solute concentration suggests that the relative discrepancy between the conditional concentration profiles that one can observe in the domain across a collection of Monte Carlo realizations tends to decrease as a consequence of the heterogeneity of the dispersion properties of the two media.

\section{Acknowledgements}

The authors would like to thank the EU and MIUR for funding, in the frame of the collaborative international Consortium (WE-NEED) financed under the ERA-NET WaterWorks2014 Cofunded Call. This ERANET is an integral part of the 2015 Joint Activities developed by the Water Challenges for a Changing World Joint Programme Initiative (Water JPI). Part of the work was developed while Prof. P. Ackerer was Invited Professor at Politecnico di Milano (Department of Civil and Environmental Engineering).

\section{Supplementary materials}

Supplementary material associated with this article can be found, in the online version, at doi:10.1016/j.advwatres.2019.04.005.

\section{Appendix A - Cross-covariance between dispersivity and concentration, $\left.\left\langle\alpha^{\prime}{ }_{L_{i}} C_{x}\right\rangle\right|_{\beta}$}

The equation satisfied by the (conditional) cross-correlation terms $\left.\left\langle\alpha^{\prime}{ }_{L_{i}} C_{x}\right\rangle\right|_{\beta}$ (with $i=1,2$ ) appearing in (11) can be obtained by multiplying (7) by $\alpha_{L_{i}}^{\prime}$ and considering the second of (9) as

$$
\begin{aligned}
& \frac{\left.\partial\left\langle\alpha^{\prime}{ }_{L_{i}} C_{x}\right\rangle\right|_{\beta}}{\partial t}+\frac{\left.\partial\left\langle\alpha^{\prime}{ }_{L_{i}} C_{x}\right\rangle\right|_{\beta}}{\partial x}-\frac{\partial}{\partial x}\left(\left[\left.H(\beta-x) \frac{\partial}{\partial x}\left\langle\alpha_{L_{1}} \alpha^{\prime}{ }_{L_{i}} C_{x}\right\rangle\right|_{\beta}\right.\right. \\
& \left.\left.+\left.H(x-\beta) \frac{\partial}{\partial x}\left\langle\alpha_{L_{2}} \alpha^{\prime}{ }_{L_{i}} C_{x}\right\rangle\right|_{\beta}\right]\right)=0
\end{aligned}
$$

subject to the initial and boundary conditions

$$
\left.\left\langle\alpha^{\prime}{ }_{L_{i}} C_{x}\right\rangle\right|_{\beta}=0 \quad \text { for } 0 \leq x \leq 1, t=0
$$

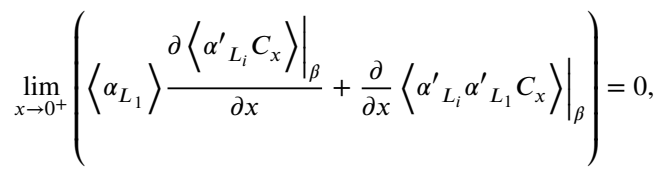

$$
\begin{aligned}
& \frac{\left.\partial\left\langle\alpha^{\prime}{ }_{L_{i}} C_{x}\right\rangle\right|_{\beta}}{\partial x}=0 \text { for } x=1, \forall t>0
\end{aligned}
$$

The zero-order component $\left.\left\langle\alpha^{\prime}{ }_{L_{i}} C_{x}\right\rangle\right|_{\beta} ^{[0]}$ (with $i=1,2$ ) vanishes, while the second-order contribution $\left.\left\langle\alpha^{\prime}{ }_{L_{i}} C_{x}\right\rangle\right|_{\beta} ^{[2]}$ in (14) is evaluated from (A.1) as

$$
\begin{gathered}
\frac{\left.\partial\left\langle\alpha^{\prime}{ }_{L_{i}} C_{x}\right\rangle\right|_{\beta} ^{[2]}}{\partial t}+\frac{\left.\partial\left\langle\alpha^{\prime}{ }_{L_{i}} C_{x}\right\rangle\right|_{\beta} ^{[2]}}{\partial x} \\
-\frac{\partial}{\partial x}\left[\left(H(\beta-x)\left\langle\alpha_{L_{1}}\right\rangle+H(x-\beta)\left\langle\alpha_{L_{2}}\right\rangle\right) \frac{\left.\partial\left\langle\alpha^{\prime}{ }_{L_{i}} C_{x}\right\rangle\right|_{\beta} ^{[2]}}{\partial x}\right] \\
=\frac{\partial}{\partial x}\left[H(\beta-x) \sigma_{\alpha_{L_{i}}}^{2} \frac{\left.\partial\left\langle C_{x}\right\rangle\right|_{\beta} ^{[0]}}{\partial x}\right)
\end{gathered}
$$

subject to the initial and boundary conditions

$$
\begin{aligned}
& \left.\left\langle\alpha^{\prime}{ }_{L_{i}} C_{x}\right\rangle\right|_{\beta} ^{[2]}=0 \text { for } 0 \leq x \leq 1, \quad t=0 \\
& \lim _{x \rightarrow 0^{+}}\left(\left\langle\alpha_{L_{1}}\right\rangle \frac{\left.\partial\left\langle\alpha^{\prime}{ }_{L_{i}} C_{x}\right\rangle\right|_{\beta} ^{[2]}}{\partial x}+(2-i) \sigma_{\alpha_{L_{1}}}^{2} \frac{\left.\partial\left\langle C_{x}\right\rangle\right|_{\beta} ^{[0]}}{\partial x}\right)=0 \\
& \frac{\left.\partial\left\langle{\alpha^{\prime}}_{L_{i}} C_{x}\right\rangle\right|_{\beta} ^{[2]}}{\partial x}=0 \text { for } x=1, \forall t>0
\end{aligned}
$$

where $\sigma_{\alpha_{L_{i}}}^{2}$ is the variance of $\alpha_{L_{i}}$.

Appendix B - Solute concentration covariance, $\left.\left\langle C^{\prime}{ }_{\xi} C^{\prime}{ }_{x}\right\rangle\right|_{\beta}$

To evaluate the conditional concentration covariance, we write the equation satisfied by $\left.C_{x}^{\prime}\right|_{\beta}=\left.C_{x}\right|_{\beta}-\left.\left\langle C_{x}\right\rangle\right|_{\beta}$ upon subtracting (11) from (7) as

$$
\begin{gathered}
\frac{\left.\partial C^{\prime}{ }_{x}\right|_{\beta}}{\partial t}+\frac{\left.\partial C^{\prime}{ }_{x}\right|_{\beta}}{\partial x}-\frac{\partial}{\partial x}\left(H(\beta-x)\left(\alpha^{\prime}{ }_{L_{1}} \frac{\left.\partial\left\langle C_{x}\right\rangle\right|_{\beta}}{\partial x}+\alpha_{L_{1}} \frac{\left.\partial{C^{\prime}}^{\prime}\right|_{\beta}}{\partial x}-\frac{\left.\partial\left\langle\alpha^{\prime}{ }_{L_{1}} C_{x}\right\rangle\right|_{\beta}}{\partial x}\right)\right. \\
\left.+H(x-\beta)\left(\alpha^{\prime}{ }_{L_{2}} \frac{\left.\partial\left\langle C_{x}\right\rangle\right|_{\beta}}{\partial x}+\alpha_{L_{2}} \frac{\left.\partial{C^{\prime}}^{\prime}\right|_{\beta}}{\partial x}-\frac{\left.\partial\left\langle\alpha^{\prime}{ }_{L_{2}} C_{x}\right\rangle\right|_{\beta}}{\partial x}\right)\right)=0
\end{gathered}
$$

subject to the initial and boundary conditions

$$
\begin{aligned}
& \left.C^{\prime}{ }_{x}\right|_{\beta}=0 \quad \text { for } 0 \leq x \leq 1, \quad t=0 \\
& \lim _{x \rightarrow 0^{+}}\left\langle\alpha_{L_{1}}\right\rangle \frac{\left.\partial C^{\prime}{ }_{x}\right|_{\beta}}{\partial x}+\alpha_{L_{1}}{ }^{\prime} \frac{\left.\partial\left\langle C_{x}\right\rangle\right|_{\beta}}{\partial x}=0, \frac{\left.\partial C^{\prime}{ }_{x}\right|_{\beta}}{\partial x}=0 \quad \text { for } x=1, \forall t>0
\end{aligned}
$$

Multiplying (B.1)-(B.2) by concentration perturbation at space-time location $(\xi, \tau),\left.C_{\xi}^{\prime}\right|_{\beta}$, and applying the second of (9), one obtains the equation satisfied by the conditional concentration covariance, as given by (16)-(17).

\section{Appendix C - Numerical technique for the solution of the Moment Equations}

All equations satisfied by the conditional moments illustrated in Section 2.2.1 and in Appendices A and B have the same format. Their 
solution requires an accurate scheme with very limited numerical diffusion, especially in light of concentration variations taking place around the geomaterials' interface (see Fig. 1a-c as an example). We adapted the numerical methods and strategy developed by Siegel et al. (1997) to solve numerically the following system:

$$
\begin{aligned}
& \frac{\partial \psi}{\partial t}+\frac{\partial}{\partial x}\left(\psi-\alpha_{\psi} \frac{\partial \psi}{\partial x}\right)=Q_{\psi} \\
& \left\{\begin{array}{l}
\psi \\
\text { for } 0 \leq x \leq 1, \quad t=0
\end{array}\right. \\
& \lim _{x \rightarrow 0^{+}}-\alpha_{\psi} \frac{\partial \psi}{\partial x}=0, \quad \frac{\partial \psi}{\partial x}=0 \quad \text { for } x=1, \quad \forall t>0
\end{aligned}
$$

Here, $\alpha_{\psi}$ and $Q_{\psi}$ respectively are the (dimensionless) dispersivity and source term associated with the equation satisfied by generic quantity $\psi$. Note that in (C.1), the initial pulse injection (when needed) is embedded in $Q_{\psi}$ and the specifics of the boundary condition at $x=0$ is treated in Section 2.2.1 for each given quantity $\psi$ of interest in our study.

To identify and apply the most suited numerical method to solve the advective (hyperbolic) and dispersive components, the transport equation is split in two parts,

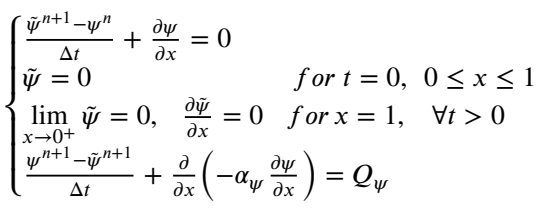

where $\tilde{\psi}$ is the solution of the advective problem and $n$ is the current time step. The hyperbolic part is solved by discontinuous Galerkin finite element (Cockburn and Shu, 1998; Di Pietro and Ern, 2012) while the dispersive part is solved by classical finite differences.

Numerical solution of the advective part of the transport equation

The spatial domain is discretized into $N_{E}$ elements of uniform width $\Delta x$, the temporal simulation window being discretized across $N_{T}$ uniform time steps, of length $\Delta t$. We treat $\psi$ in each element $E$ as a linear combination of two nodal values, i.e.,

$\tilde{\psi}(x, t)=\omega_{i}(x) \tilde{\psi}_{i}(t)+\omega_{i+1}(x) \tilde{\psi}_{i+1}(t) \quad$ for $\quad x \in\left[x_{i}, x_{i+1}\right]$

$\omega_{i}=\frac{x_{i+1}-x}{\Delta x} ; \omega_{i+1}=\frac{x-x_{i}}{\Delta x} ; \quad \Delta x=x_{i+1}-x_{i}$.

Here, $\left(\omega_{i}, \omega_{i+1}\right)$ are linear basis functions defined over element $E ; \tilde{\psi}_{i}$ and $\tilde{\psi}_{i+1}$ are the nodal approximations of $\tilde{\psi}$; and $\left(x_{i}, x_{i+1}\right)$ are the spatial coordinates of the two nodes delimiting $E$. Similar to the finite element method, the hyperbolic equation is written in a variational form, using $\left(\omega_{i}, \omega_{i+1}\right)$ as weighting functions, leading to

$$
\begin{aligned}
\int_{E} \frac{\partial \tilde{\psi}}{\partial t} \omega_{i} d x & =-\int_{E} \frac{\partial}{\partial x}(\tilde{\psi}) \omega_{i} d x=\int_{E} \tilde{\psi} \frac{\partial \omega_{i}}{\partial x} d x-\left[\tilde{\psi}_{i+1} \omega_{i}\left(x_{i+1}\right)-\tilde{\psi}_{i} \omega_{i}\left(x_{i}\right)\right] \\
& =\int_{E} \tilde{\psi} \frac{\partial \omega_{i}}{\partial x} d x+\tilde{\psi}_{i}
\end{aligned}
$$

Using the approximation of $\tilde{\psi}$ defined by Eq. (C.3) and the definition of the interpolation functions (C.4), (C.5) becomes:

$\frac{\Delta x}{6}\left[2 \frac{\partial \widetilde{\psi}_{i}}{\partial t}+\frac{\partial \widetilde{\psi}_{i+1}}{\partial t}\right]=-\frac{1}{2}\left(\widetilde{\psi}_{i}+\widetilde{\psi}_{i+1}\right)+\widetilde{\psi}_{i}$

Similarly, using $\omega_{i+1}$ as weigthing function leads to:

$$
\frac{\Delta x}{6}\left[\frac{\partial \widetilde{\psi}_{i}}{\partial t}+2 \frac{\partial \widetilde{\psi}_{i+1}}{\partial t}\right]=\frac{1}{2}\left(\widetilde{\psi}_{i}+\widetilde{\psi}_{i+1}\right)-\widetilde{\psi}_{i+1}
$$

The time discretization of the spatial derivative is split in two parts to improve accuracy and reduce numerical diffusion (Siegel et al., 1997). The first step consists in computing the variable at time $(n+1 / 2)$ using half of the time step length and an explicit time scheme. Eqs. (C.6) and (C.7) become

$\left\{\begin{array}{l}2 \widetilde{\psi}_{i}^{n+1 / 2}+\widetilde{\psi}_{i+1}^{n+1 / 2}=\left(2+\frac{3}{2} \kappa\right) \widetilde{\psi}_{i}^{n}+\left(1-\frac{3}{2} \kappa\right) \widetilde{\psi}_{i+1}^{n} \\ \widetilde{\psi}_{i}^{n+1 / 2}+2 \widetilde{\psi}_{i+1}^{n+1 / 2}=\left(1+\frac{3}{2} \kappa\right) \widetilde{\psi}_{i}^{n}+\left(2-\frac{3}{2} \kappa\right) \widetilde{\psi}_{i+1}^{n}\end{array}\right.$ where $\kappa=\Delta t / \Delta x$ is the Courant number. For the second step, Eqs. (C.6) and (C.7) become

$$
\left\{\begin{array}{l}
2 \widetilde{\psi}_{i}^{n+1}+\widetilde{\psi}_{i+1}^{n+1}=2 \widetilde{\psi}_{i}^{n}+\widetilde{\psi}_{i}^{n}-3 \kappa\left(\widetilde{\psi}_{i}^{n+1 / 2}+\widetilde{\psi}_{i+1}^{n+1 / 2}\right)+6 \kappa\left(\widetilde{\psi}_{i}^{n+1 / 2}\right)^{*} \\
\widetilde{\psi}_{i}^{n+1}+2 \widetilde{\psi}_{i+1}^{n+1}=\widetilde{\psi}_{i}^{n}+2 \widetilde{\psi}_{i}^{n}+3 \kappa\left(\widetilde{\psi}_{i}^{n+1 / 2}+\widetilde{\psi}_{i+1}^{n+1 / 2}\right)-6 \kappa\left(\widetilde{\psi}_{i+1}^{n+1 / 2}\right)^{*}
\end{array}\right.
$$

The last terms $\left(\widetilde{\psi}_{i}^{n+1 / 2}, \widetilde{\psi}_{i+1}^{n+1 / 2}\right)$ in both equations of system (C.9) represent the variable at location $x_{i}$ and $x_{i+1}$, respectively. At $x_{i}$, (and respectively at $x_{i+1}$ ), there are two possible values for $\widetilde{\psi}_{i}^{n+1 / 2}$ (respectively $\widetilde{\psi}_{i+1}^{n+1 / 2}$ ), one defined for element $E$ and the other defined at the previous (respectively next) element. The selected value is the upstream one, i.e., assuming that the flow direction coincides with the positive direction of the $x$-axis, we have

$\left\{\begin{array}{l}\left(\widetilde{\psi}_{i}^{n+1 / 2}\right)^{*}=\widetilde{\psi}_{i+1, E-1}^{n+1 / 2} \\ \left(\widetilde{\psi}_{i+1}^{n+1 / 2}\right)^{*}=\widetilde{\psi}_{i+1}^{n+1 / 2}\end{array}\right.$

Therefore, the solution of the advective part is obtained by solving the following system of equations

$\left\{\begin{array}{l}2 \widetilde{\psi}_{i}^{n+1}+\widetilde{\psi}_{i+1}^{n+1}=2 \widetilde{\psi}_{i}^{n}+\widetilde{\psi}_{i}^{n}-3 \kappa\left(\widetilde{\psi}_{i}^{n+1 / 2}+\widetilde{\psi}_{i+1}^{n+1 / 2}\right)+6 \kappa \widetilde{\psi}_{i+1, E-1}^{n+1 / 2} \\ \widetilde{\psi}_{i}^{n+1}+2 \widetilde{\psi}_{i+1}^{n+1}=\widetilde{\psi}_{i}^{n}+2 \widetilde{\psi}_{i}^{n}+3 \kappa\left(\widetilde{\psi}_{i}^{n+1 / 2}+\widetilde{\psi}_{i+1}^{n+1 / 2}\right)-6 \kappa \widetilde{\psi}_{i+1}^{n+1 / 2}\end{array}\right.$

The stability of the solution is improved by a slope limiting procedure (Hoteit et al., 2004). The latter consists in reducing the variation of the target variable within the element to provide physically acceptable solutions. This procedure does not change the mass inside the element, and, therefore, does not negatively affect mass balance over the domain. The local values of $\tilde{\psi}$ are changed following

$\left\{\begin{array}{l}\min \left(\tilde{\psi}_{E-1}^{n+1}, \tilde{\psi}_{E}^{n+1}\right) \leq \widetilde{\psi}_{i}^{n+1} \leq \max \left(\tilde{\psi}_{E-1}^{n+1}, \tilde{\psi}_{E}^{n+1}\right) \\ \min \left(\tilde{\psi}_{E}^{n+1}, \tilde{\psi}_{E+1}^{n+1}\right) \leq \widetilde{\psi}_{i+1}^{n+1} \leq \max \left(\tilde{\psi}_{E}^{n+1}, \tilde{\psi}_{E+1}^{n+1}\right)\end{array}\right.$

where $\tilde{\psi}_{E}^{n+1}$ is the average of $\tilde{\psi}^{n+1}$ in element $E$, i.e.,

$\tilde{\psi}_{E}^{n+1}=\frac{1}{2}\left(\tilde{\psi}_{i}^{n+1}+\tilde{\psi}_{i+1}^{n+1}\right)$

The solution is stable for a Courant number $\kappa=\Delta t / \Delta x<1.0$.

Numerical solution of the dispersive part of the transport equation

The second equation of system (C.2) is solved with a standard finite differences formulation using an implicit time scheme which leads to

$\Delta x \frac{\psi_{E}^{n+1}-\widetilde{\psi}_{E}^{n+1}}{\Delta t}=\alpha_{+}^{E} \frac{\psi_{E+1}^{n+1}-\psi_{E}^{n+1}}{\Delta x}-\alpha_{-}^{E} \frac{\psi_{E}^{n+1}-\psi_{E-1}^{n+1}}{\Delta x}+\frac{Q_{E}^{n+1}+Q_{E}^{n}}{2}$

with

$\left\{\begin{array}{l}\alpha_{-}^{E}=\frac{2 \alpha_{E-1} \alpha_{E}}{\alpha_{E-1}+\alpha_{E}} \\ \alpha_{+}^{E}=\frac{2 \alpha_{E} \alpha_{E+1}}{\alpha_{E}+\alpha_{E+1}}\end{array}\right.$

The inter-element equivalent dispersivities $\left(\alpha_{-}^{E}, \alpha_{+}^{E}\right)$ are the harmonic mean of the element dispersivities. Such an harmonic mean is obtained by fulfilling the requirement of continuity for state variables and flux between two elements.

Considering the boundary conditions described in Eq. (C.1) and rewriting Eq. (C.14) leads to the following system of equations

$\left\{\begin{array}{l}\left(1+\frac{\kappa \alpha_{+}^{1}}{\Delta x}\right) \psi_{1}^{n+1}-\left(\frac{\kappa \alpha_{+}^{1}}{\Delta x}\right) \psi_{2}^{n+1}=\widetilde{\psi}_{1}^{n+1}+\frac{\Delta t}{\Delta x} \frac{Q_{1}^{n+1}+Q_{1}^{n}}{2} \\ \left(-\frac{\kappa \alpha_{-}^{E}}{\Delta x}\right) \psi_{E-1}^{n+1}+\left(1+\frac{\kappa \alpha_{-}^{E}}{\Delta x}+\frac{\kappa \alpha_{+}^{E}}{\Delta x}\right) \psi_{E}^{n+1}-\left(\frac{\kappa \alpha_{+}^{E}}{\Delta x}\right) \psi_{E+1}^{n+1}=\widetilde{\psi}_{E}^{n+1} \\ \quad+\frac{\Delta t}{\Delta x} \frac{Q_{E}^{n+1}+Q_{E}^{n}}{2} E=2 \ldots N_{E-1} \\ \left(-\frac{\kappa \alpha_{-} E}{\Delta x}\right) \psi_{N_{E-1}}^{n+1}+\left(1+\frac{\kappa \alpha_{-} N_{E}}{\Delta x}\right) \psi_{N_{E}}^{n+1}=\widetilde{\psi}_{N_{E}}^{n+1}+\frac{\Delta t}{\Delta x} \frac{Q_{N_{E}}^{n+1}+Q_{N_{E}}^{n}}{2}\end{array}\right.$

This tri-diagonal system is solved by a standard LU decomposition (Thomas algorithm). 


\section{References}

Appuhamillage, T.A., Bokil, V.A., Thomann, E., Waymire, E., Wood, B.D., 2010. Solute transport across an interface: a Fickian theory for skewness in breakthrough curves. Water Resour. Res. 46, W07511.

Alvarez-Ramirez, J., Valdes-Parada, F.J., Rodriguez, E., Dagdug, L., Inzunza, L., 2014. Asymmetric transport of passive tracers across heterogeneous porous media. Physica.

Berkowitz, B., Cortis, A., Dror, I., Scher, H., 2009. Laboratory experiments on dispersive transport across interfaces: the role of flow direction. Water Resour. Res. 45, W02201.

Berkowitz, B., Cortis, A., Dentz, M., Scher, H., 2006. Modeling non-Fickian transport in geological formations as a continuous time random walk. Rev. Geophys. 44.

Boso, F., Tartakovsky, D.M., 2016. The method of distributions for dispersive transport in porous media with uncertain hydraulic properties. Water Resour. Res. 52, 4700-4712.

de Barros, F.P.J., Fiori, A., 2014. First-order based cumulative distribution function for solute concentration in heterogeneous aquifers: theoretical analysis and implications for human health risk assessment. Water Resour. Res. 50, 4018-4037.

de Barros, F.J.P., Dentz, M., 2016. Pictures of blockscale transport: effective versus ensemble dispersion and its uncertainty. Adv. Water Resour. 91, 11-22.

de Barros, F.J.P., Rubin, Y., 2011. Modelling block-scale macrodispersion as a random space function. J. Fluid Mech. 676, 514-545.

Chaudhuri, A., Sekhar, M., 2005. Analytical solutions for macrodispersion in a 3D heterogeneous porous media with random hydraulic conductivity and dispersivity. Transp. Porous Med. 58, 217-241.

Cockburn, B., Shu, C.W., 1998. The local discontinuous Galerkin finite element method for convection-diffusion systems. SIAM J. Numer. Anal. 35, 2440-2463.

Coelho, D., Thovert, J.F., Adler, P.M., 1997. Geometrical and transport properties of random packings of spheres and aspherical particles. Phys. Rev. E 55 (2), 1959-1978.

Cortis, A., Zoia, A., 2009. Model dispersive transport across sharp interfaces between porous materials. Phys. Rev. E 80, 011122.

Cushman, J.H., Ginn, T.R., 1993. Nonlocal dispersion in media with continuously evolving scales of heterogeneity. Transp. Porous Med. 13, 123-138.

Dentz, M., Tartakovsky, D.M., 2010. Probability density functions for passive scalars dispersed in random velocity fields. Geophys. Res. Lett. 37, L24406.

Di Pietro, D.A., Ern, A., 2012. Mathematical Aspects of Discontinuous Galerkin Methods. Springer, p. 384.

Fernàndez-Garcia, D., Llerar-Meza, G., Gómez-Hernàndez, J.J., 2009. Upscaling transport with mass transfer models: mean behavior and propagation of uncertainty. Water Resour. Res. 45, W10411. https://doi.org/10.1029/2009WR007764.

Fiori, A., Zarlenga, A., Jankovic, I., Dagan, G., 2017. Solute transport in aquifers: the comeback of the advection dispersion equation and the first order approximation. Adv. Water Resour. 110, 349-359.

Ghanem, R.G., Spanos, P., 1991. Stochastic Finite elements: a Spectral Approach. Springer.

Gelhar, L.W., Axness, C.L., 1983. Three-dimensional stochastic analysis of macrodispersion in aquifers. Water Resour. Res. 19 (1), 161-180.

Gist, G.A., Thompson, A.H., Katz, A.J., Higgins, R.L., 1990. Hydrodynamic dispersion and pore geometry in consolidated rock. Phys. Fluids 1533-1544.

Godoy, V.A., Zuquette, L.V., Gómez-Hernàndez, J.J., 2018. Stochastic upscaling of hydrodynamic dispersion and retardation factor in a physically and chemically heterogeneous tropical soil. Stoch. Environ. Res. Risk Assess. https://doi.org/10.1007/s00477-018-1624-z.

Gramling, C.M., Harvey, C.F., Meigs, L.C., 2002. Reactive transport in porous media: a comparison of model prediction with laboratory visualization. Environ. Sci. Technol. $36,2508-2514$

Guadagnini, A., Guadagnini, L., Tartakovsky, D.M., Winter, C.L., 2003. Random domain decomposition for flow in heterogeneous stratified aquifers. Stoch. Environ. Res. Risk Assess. 17, 394-407.
Hoteit, H., Ackerer, P., Mosé, R., Erhel, J., Philippe, B., 2004. New two-dimensional slope limiters for discontinuous Galerkin methods on arbitrary meshes. Int. J. Numer. Methods Eng. 61 (14), 2566-2593.

Kitanidis, P.K., 2017. Teaching and communicating dispersion in hydrogeology, with emphasis on the applicability of the Fickian model. Adv. Water Res. 106, 11-23.

Kuo, R.-K.H., Irwin, C., Greenkorn, R.A., Cushman, J.H., 1999. Experimental investigation of mixing in aperiodic heterogeneous porous media: comparison with stochastic transport theory. Transp. Porous Med. 37, 169-182.

Le Borgne, T., Dentz, M., Bolster, D., Carrera, J., de Dreuzy, J-R., Davy, P., 2010. Non-Fickian mixing: temporal evolution of the scalar dissipation rate in porous media. Adv. Water Resour 33, 1468-1475.

Levy, M., Berkowitz, B., 2003. Measurement and analysis of non-Fickian dispersion in heterogeneous porous media. J. Contam. Hydrol. 64, 203-226.

Li, H., Zhang, D., 2007. Probabilistic collocation method for flow in porous media: comparison with other stochastic methods. Water. Resour. Res. 43, W09409.

Leij, F.J., van Genuchten, M.T., 1995. Approximate analytical solutions for solute transport in two-layer porous media. Transp. Porous Media 18, 65-85.

Morales, C.E., Neuman, S.P., Guadagnini, A., 2006a. Nonlocal and localized analyses of nonreactive solute transport in bounded randomly heterogeneous porous media: theoretical framework. Adv. Water Resour. 29, 1238-1255.

Morales, C.E., Neuman, S.P., Guadagnini, A., 2006b. Nonlocal and localized analyses of nonreactive solute transport in bounded randomly heterogeneous porous media: computational analysis. Adv. Water Resour. 29, 1399-1418.

Moslehi, M., de Barros, F.P.J., Ebrahimi, F., Sahimi, M., 2016. Upscaling of solute transport in disordered porous media by wavelet transformations. Adv. Water Resour. 96, $180-189$.

Neuman, S.P., 1993. Eulerian-Lagrangian theory of transport in space-time nonstationary velocity fields: exact nonlocal formalism by conditional moments and weak approximations. Water Resour. Res. 29 (3), 633-645.

Porta, G., Bijeljic, B., Blunt, M.J., Guadagnini, A., 2015. Continuum-scale characterization of solute transport based on pore-scale velocity distributions. Geophys. Res. Lett. 42 (18), 7537-7545.

Rubin, Y., 2003. Applied Stochastic Hydrogeology. Oxford.

Siegel, P., Mosé, R., Ackerer, P., Jaffre, J., 1997. Solution of the advection-diffusion equation using a combination of discontinuous and mixed finite elements. Int. J. Numer. Methods Fluids 24 (6), 595-613.

Sternberg, S.P.K., 2004. Dispersion measurements in highly heterogeneous laboratory scale porous media. Transp. Porous Med. 54, 107-124.

Tartakovsky, D.M., Guadagnini, A., 2004. Effective properties of random composites SIAM J. Sci. Comp. 26 (2), 625-635.

Vishal, V., Leung, J.Y., 2018. Statistical scale-up of 3D particle-tracking simulation for non-Fickian dispersive solute transport modeling. Stoch. Environ. Res. Risk Assess. 32, 2075-2091.

Winter, C.L., Tartakovsky, D.M., Guadagnini, A., 2002. Numerical solutions of moment equations for flow in heterogeneous composite aquifers. Water Resour. Res. 38 (5).

Winter, C.L., Tartakovsky, D.M., 2000. Mean flow in composite porous media. Geophys. Res. Lett. 27 (12), 1759-1762.

Woods, B.D., Cherblanc, F., Quintard, M., Whitaker, S., 2003. Volume averaging for determining the effective dispersion tensor: closure using periodic unit cells and comparison with ensemble averaging. Water Resour. Res. 39 (8), 1210.

Xu, Z., Meakin, P., 2013. Upscaling of solute transport in heterogeneous media with non-uniform flow and dispersion fields. Appl. Math. Modell. 37, 8533-8542.

Zech, A., Attinger, S., Cvetkovic, V., Dagan, G., Dietrich, P., Fiori, A., Rubin, Y., Teutsch, G., 2015. Is unique scaling of aquifer macrodispersivity supported by field data? Water Resour. Res. 51, 7662-7679.

Zhang, X., Qi, X., Qiao, D., 2010. Change in macroscopic concentration at the interface between different materials: continuous or discontinuous. Water Resour. Res. 46, W10540. 